\title{
Standard Model parton distributions at very high energies
}

\author{
Christian W. Bauer, ${ }^{a, b}$ Nicolas Ferland ${ }^{a}$ and Bryan R. Webber ${ }^{c}$ \\ ${ }^{a}$ Ernest Orlando Lawrence Berkeley National Laboratory, University of California, \\ Berkeley, CA 94720, U.S.A. \\ ${ }^{b}$ Theoretical Physics Department, CERN, \\ Geneva, Switzerland \\ ${ }^{c}$ University of Cambridge, Cavendish Laboratory, \\ J.J. Thomson Avenue, Cambridge, U.K. \\ E-mail: cwbauer@lbl.gov, nferland@lbl.gov, webber@hep.phy.cam.ac.uk
}

ABSTRACT: We compute the leading-order evolution of parton distribution functions for all the Standard Model fermions and bosons up to energy scales far above the electroweak scale, where electroweak symmetry is restored. Our results include the 52 PDFs of the unpolarized proton, evolving according to the $\mathrm{SU}(3), \mathrm{SU}(2), \mathrm{U}(1)$, mixed $\mathrm{SU}(2) \times \mathrm{U}(1)$ and Yukawa interactions. We illustrate the numerical effects on parton distributions at large energies, and show that this can lead to important corrections to parton luminosities at a future $100 \mathrm{TeV}$ collider.

KEYWORDs: Jets, QCD Phenomenology

ARXIV EPRINT: 1703.08562 


\section{Contents}

1 Introduction 1

2 The evolution of parton distributions in the full Standard Model 3

2.1 Definition of the parton distribution functions 3

2.2 General evolution equations 6

$\begin{array}{ll}2.3 \text { Splitting functions } & 10\end{array}$

$\begin{array}{lll}2.4 & \text { Running couplings } & 10\end{array}$

$2.5 I=3: \mathrm{SU}(3)$ interactions 11

$\begin{array}{lll}2.6 & I=1: \mathrm{U}(1) \text { interactions } & 12\end{array}$

$2.7 \quad I=2: \mathrm{SU}(2)$ interactions 13

$2.8 I=Y$ : Yukawa interactions $\quad 15$

$\begin{array}{lll}2.9 & I=M: \text { mixed } B-W_{3} \text { interactions } & 15\end{array}$

3 Implementation details $\quad \mathbf{1 6}$

$\begin{array}{lll}3.1 & \text { Switching to a basis of conserved quantum numbers } & 17\end{array}$

$\begin{array}{ll}3.2 & \text { Cancellation of double-logarithmic dependence in evolution equations } \\ & 18\end{array}$

$\begin{array}{llr}4 & \text { Results } & 19\end{array}$

5 Conclusions $\quad 26$

$\begin{array}{ll}\text { A Equations used in the forward evolution } & 27\end{array}$

$\begin{array}{lll}\text { A.1 } & \mathrm{SU}(3) \text { interaction } & 27\end{array}$

$\begin{array}{lll}\text { A.2 } & \mathrm{U}(1) \text { interaction } & 27\end{array}$

A.3 $\mathrm{SU}(2)$ interaction 28

$\begin{array}{ll}\text { A.4 Yukawa interaction } & 29\end{array}$

$\begin{array}{lll}\text { A.5 Mixed interaction } & 30\end{array}$

\section{Introduction}

Experiments at the Large Hadron Collider are now probing the structure of matter at scales comparable with, and even beyond, the characteristic scale of electroweak symmetry breaking. So far, no evidence has been found for a breakdown of the Standard Model (SM) in particle collisions. Indeed, there is a logical possibility that the SM remains a good description of hard scattering processes up to scales far beyond those of any conceivable particle colliders. It is therefore of interest to examine the features predicted by the SM for collider events well above the electroweak scale. For this purpose, Monte Carlo event 
generators including all the SM interactions on an equal footing are necessary. Such generators would be useful for investigating the limits of LHC searches, the potential of possible future colliders and cosmic processes at ultrahigh energies.

To construct a general-purpose SM event generator, ${ }^{1}$ the three phases of a hard collision, namely initial-state parton showering, parton-parton collision and final-state showering, need to be simulated including all SM particles and interactions. For the initial-state showering, parton distribution functions (PDFs) for all the SM fermions and bosons need to be computed and tabulated beforehand, so that showering can be generated backwards from the hard process, guided by the scale dependence of the PDFs $[2,3]$.

Recently, a final-state parton shower including emissions from all interactions in the Standard Model was developed [4], which illustrated the importance of electroweak splittings at high energies. For initial-state radiation the generalization of the DGLAP [5-7] evolution equations using all the Standard Model interactions has been worked out in [8], but so far no numerical implementation of these results has been published.

As already mentioned, understanding the DGLAP evolution of PDFs using all interactions of the SM is a required first step in developing a complete initial state parton shower. Moreover, it already allows us to study many new qualitative features of very high-energy processes, such as lepton-initiated processes in hadron collisions and the polarization induced by electroweak PDF evolution.

The inclusion of QED corrections into parton distributions is a well established procedure [9-16]. However, above the electroweak scale around $100 \mathrm{GeV}$, the contributions of other electroweak bosons become non-negligible and new effects appear [8, 17-30]. PDFs of leptons, vector and scalar bosons are generated dynamically, and left- and right-handed fermions evolve differently. There are also comparable effects in the third generation of quarks due to their Yukawa interactions. Some effects of the SU(2) interaction are doublelogarithmically enhanced, due to the non-singlet nature of the incoming states.

The PDF evolution equations for the full Standard Model have been presented in ref. [8]. In the present paper we recast those equations in a form suitable for event generation and solve them numerically for a given set of input distributions at the electroweak scale. The resulting PDF set extends through the region of interest for future colliders and well beyond, so that we can study the onset of the regime where all the SM interactions start to become comparable.

Our solutions to the SM evolution equations are obtained in the approximation of exact $\mathrm{SU}(3) \times \mathrm{SU}(2) \times \mathrm{U}(1)$ symmetry. That is, we neglect fermion and Higgs masses and the Higgs vacuum expectation value, the effects of these being power-suppressed at high scales. We impose an infra-red cutoff $m_{V}$ on interactions that involve the emission of an electroweak vector boson, $V=W^{i}$ for $\mathrm{SU}(2)$ or $B$ for $\mathrm{U}(1)$. Leading-order evolution kernels and one-loop running couplings are used. All the electroweak PDFs are generated dynamically from the QCD plus photon PDFs, starting from a matching scale $q_{0} \sim m_{V}$. In practice we take $q_{0}=m_{V}=100 \mathrm{GeV}$. In section 4 we show some effects of varying these parameters, to provide an indication of uncertainties due to subleading logarithms and power-suppressed terms.

\footnotetext{
${ }^{1}$ For a review of existing generators, see ref. [1].
} 
For the evolution of the photon, we decompose its PDF into $W^{3}, B$ and mixed $B / W^{3}$ components at the input scale, evolve these components, and reconstruct the photon PDF from them at higher scales using the running $\mathrm{SU}(2)$ and $\mathrm{U}(1)$ couplings. For the top quark, we set the PDF to zero below the top mass scale and then use the leading-order massless evolution kernels, as for other fermions. This treatment of the transition region around the electroweak scale is clearly over-simplified but it should give a reliable indication of the magnitude of electroweak effects at higher energies.

The accuracy of our resulting PDFs is leading logarithmic, with subleading logarithmic effects included where possible, but not in a complete way. Contributions to the evolution from the $\mathrm{U}(1), \mathrm{SU}(3)$ and Yukawa interactions are therefore correct at the single logarithmic level. However, as mentioned above, the $\mathrm{SU}(2)$ interactions give rise to double logarithmic effects in the PDF evolution, such that single logarithmic effects in $\mathrm{SU}(2)$ non-singlet quantities are not fully under control.

The organization of the paper is as follows. In section 2 we define the relevant parton distribution functions for unpolarized proton beams and the general form of their evolution equations, paying particular attention to the conservation of momentum in the presence of the cutoff $m_{V}$ for vector boson emission. After specifying all the necessary splitting functions and running couplings, we write the explicit evolution equations associated with the five interactions: $\mathrm{SU}(3), \mathrm{U}(1), \mathrm{SU}(2)$, Yukawa and mixed $\mathrm{U}(1) \times \mathrm{SU}(2)$, for all the SM partons in a flavor basis. As usual for DGLAP evolution, we do not include 4 point interactions which are suppressed at high energies.

For a numerical implementation, as described in section 3, the flavor basis is not convenient, as too many coupled equations are involved. Instead we use the basis of conserved quantum numbers introduced in ref. [8]. As shown there, the double-logarithmic evolution of SU(2) non-singlet PDFs can then be factored out, which stabilizes and accelerates the solution of the equations. In this way we are able to evolve all the SM PDFs to arbitrarily high scales with satisfactory speed and precision. In practice we evolve up to $10^{8} \mathrm{GeV}$, where the approach to asymptotic behavior is well established.

In section 4, we present a selection of results that illustrate the extent to which electroweak effects change the behavior of the various PDFs. In particular, we show changes in the PDFs of strongly interacting particles relative to pure QCD evolution, and show the size of the PDFs for electroweak gauge bosons relative to the gluon PDF. Finally, we present results of the associated changes in parton-parton luminosities at a $100 \mathrm{TeV} p p$ collider and show the sensitivity of our results to changes of the input values of $m_{V}$ and $q_{0}$. Our conclusions are presented in section 5 .

\section{The evolution of parton distributions in the full Standard Model}

\subsection{Definition of the parton distribution functions}

The standard definition of an $x$-weighted parton distribution is given by the matrix element of a bi-local operator, separated along the lightcone. For fermions, one finds the standard definition, but without spin averaging as we are separating the fermions into left- and 
right-handed. Thus, each fermion has only one possible spin determined by its helicity and the sign of its momentum

$$
\begin{aligned}
& f_{i}(x, \mu)=x \int \frac{d y}{2 \pi} e^{-i 2 x \bar{n} \cdot p y}\left\langle p\left|\bar{\psi}^{(i)}(y) \not h \psi^{(i)}(-y)\right| p\right\rangle, \\
& f_{\bar{i}}(x, \mu)=x \int \frac{d y}{2 \pi} e^{-i 2 x \bar{n} \cdot p y}\left\langle p\left|\psi^{(i)}(y) \not \bar{h} \bar{\psi}^{(i)}(-y)\right| p\right\rangle,
\end{aligned}
$$

where $\mu$ is the renormalization scale. Since we have separate left- and right-handed PDFs, for each generation there are a total of 8 quark PDFs and 6 lepton PDFs to consider, giving a total of 42 fermion PDFs.

Parton distributions functions of the vector bosons are given by

$$
f_{V}(x, \mu)=\left.\frac{2}{\bar{n} \cdot p} \int \frac{d y}{2 \pi} e^{-i 2 x \bar{n} \cdot p y} \bar{n}_{\mu} \bar{n}^{\nu}\left\langle p\left|V^{\mu \lambda}(y) V_{\lambda \nu}(-y)\right| p\right\rangle\right|_{\text {spin avg. }} .
$$

Since $\mathrm{SU}(3)$ is unbroken, we consider a single PDF to describe the gluon field. For the $\mathrm{SU}(2) \otimes \mathrm{U}(1)$ symmetry, on the other hand, one needs to take the symmetry breaking into account. For the $W^{+}$and $W^{-}$boson we simply include separate PDFs for each of the two gauge bosons. For the $B$ and $W_{3}$, however, one needs to be more careful to take the mixed contributions of these two bosons into account. Such contributions arise from the fact that the left-handed fermions and Higgs carry both isospin and hypercharge. This implies that besides $B$ and $W_{3}$ PDFs one needs to include a mixed PDF, which is given by ${ }^{2}$

$$
f_{B W}(x)=\left.\frac{2}{\bar{n} \cdot p} \int \frac{d y}{2 \pi} e^{-i 2 x \bar{n} \cdot p y} \bar{n}^{\mu} \bar{n}_{\nu}\left\langle p\left|B_{\mu \lambda}(y) W_{3}^{\lambda \nu}(-y)\right| p\right\rangle\right|_{\text {spin avg. }}+\text { h.c. . }
$$

From these PDFs one can then construct the PDF for the photon, the transversely-polarized $Z^{0}$ and their mixed state as a transformation of the PDF for the $B$, the $W_{3}$ and their mixed state. Using $A=c_{W} B+s_{W} W_{3}$ and $Z^{0}=-s_{W} B+c_{W} W_{3}$ one finds

$$
\left(\begin{array}{c}
f_{\gamma} \\
f_{Z} \\
f_{\gamma Z}
\end{array}\right)=\left(\begin{array}{ccc}
c_{W}^{2} & s_{W}^{2} & c_{W} s_{W} \\
s_{W}^{2} & c_{W}^{2} & -c_{W} s_{W} \\
-2 c_{W} s_{W} & 2 c_{W} s_{W} & c_{W}^{2}-s_{W}^{2}
\end{array}\right)\left(\begin{array}{c}
f_{B} \\
f_{W_{3}} \\
f_{B W}
\end{array}\right)
$$

and thus

$$
\left(\begin{array}{c}
f_{B} \\
f_{W_{3}} \\
f_{B W}
\end{array}\right)=\left(\begin{array}{ccc}
c_{W}^{2} & s_{W}^{2} & -c_{W} s_{W} \\
s_{W}^{2} & c_{W}^{2} & c_{W} s_{W} \\
2 c_{W} s_{W} & -2 c_{W} s_{W} & c_{W}^{2}-s_{W}^{2}
\end{array}\right)\left(\begin{array}{c}
f_{\gamma} \\
f_{Z} \\
f_{\gamma Z}
\end{array}\right)
$$

For the electroweak input at scale $\mu=q_{0}$ we have $f_{\gamma}\left(x, q_{0}\right) \neq 0$ and $f_{Z}\left(x, q_{0}\right)=f_{\gamma Z}\left(x, q_{0}\right)=0$, so the input conditions at that scale are

$$
f_{B}=c_{W}^{2} f_{\gamma}, \quad f_{W_{3}}=s_{W}^{2} f_{\gamma}, \quad f_{B W}=2 c_{W} s_{W} f_{\gamma} .
$$

\footnotetext{
${ }^{2}$ Note that our definition of the mixed PDF $f_{B W}$ is the sum of $B W_{3}$ and $W_{3} B$ contributions, and similarly for the mixed $\operatorname{PDF} f_{\gamma Z}$.
} 
where $s_{W}$ and $c_{W}$ depend on the value of $q$ of the PDFs

$$
\begin{aligned}
& s_{W} \equiv s_{W}(q)=\sqrt{\frac{\alpha_{1}(q)}{\alpha_{1}(q)+\alpha_{2}(q)}} \\
& c_{W} \equiv c_{W}(q)=\sqrt{\frac{\alpha_{2}(q)}{\alpha_{1}(q)+\alpha_{2}(q)}} .
\end{aligned}
$$

Thus, when relating the PDFs at the input scale $\mu=q_{0}$ in eq. (2.7), one chooses $s_{W} \equiv$ $s_{W}\left(q_{0}\right)$ and $c_{W} \equiv c_{W}\left(q_{0}\right)$. After evolving these three unbroken PDFs to a higher scale $q$, the physical photon and $Z^{0} \mathrm{PDF}$ are reconstructed using the corresponding running values of $c_{W}(q)$ and $s_{W}(q)$.

Finally, one needs to include PDFs for the scalar bosons. One writes

$$
f_{H}(x)=x \int \frac{d y}{2 \pi} e^{-i 2 x \bar{n} \cdot p y}\langle p|\Phi(y) \Phi(-y)| p\rangle,
$$

and PDFs for each of the 4 Higgs fields $H^{0}, \bar{H}^{0}, H^{+}$and $H^{-}$are included. The relationship to the 4 Higgs fields in the unbroken basis to the physical Higgs and the longitudinal gauge bosons is as follows: the $H^{ \pm}$PDFs correspond to those of the longitudinally polarized $W^{ \pm}$. In the notation of ref. [8], the neutral Higgs fields are

$$
H^{0}=\frac{\left(h-i Z_{L}\right)}{\sqrt{2}}, \quad \bar{H}^{0}=\frac{\left(h+i Z_{L}\right)}{\sqrt{2}},
$$

where $h$ and $Z_{L}$ represent the Higgs and the longitudinal $Z^{0}$ fields, respectively. The corresponding PDFs are

$$
\begin{aligned}
& f_{H^{0}}=\frac{1}{2}\left[f_{h}+f_{Z_{L}}+i\left(f_{h Z_{L}}-f_{Z_{L} h}\right)\right], \\
& f_{\bar{H}^{0}}=\frac{1}{2}\left[f_{h}+f_{Z_{L}}-i\left(f_{h Z_{L}}-f_{Z_{L} h}\right)\right],
\end{aligned}
$$

and one can also define the mixed PDFs

$$
\begin{aligned}
f_{H^{0} \bar{H}^{0}} & =\frac{1}{2}\left[f_{h}-f_{Z_{L}}-i\left(f_{h Z_{L}}+f_{Z_{L} h}\right)\right], \\
f_{\bar{H}^{0} H^{0}} & =\frac{1}{2}\left[f_{h}-f_{Z_{L}}+i\left(f_{h Z_{L}}+f_{Z_{L} h}\right)\right] .
\end{aligned}
$$

Both of these mixed PDF carry non-zero hypercharge, such that they are not produced by the DGLAP evolution in the unbroken gauge theory as considered in this paper. ${ }^{3}$ Thus, one immediately finds

$$
f_{h}-f_{Z_{L}}=f_{h Z_{L}}+f_{Z_{L} h}=0
$$

and

$$
f_{h}=f_{Z_{L}}=\frac{1}{2}\left(f_{H^{0}}+f_{\bar{H}^{0}}\right), \quad f_{h Z_{L}}=-f_{Z_{L} h}=-\frac{i}{2}\left(f_{H^{0}}-f_{\bar{H}^{0}}\right) .
$$

\footnotetext{
${ }^{3}$ They are only produced through insertions of the Higgs vacuum.
} 
In summary, there are a total of 52 parton distribution functions that need to be considered. Apart from the QCD quark and gluon distributions, the charged leptons, and the neutral electroweak boson PDFs (2.7), all the other SM PDFs are set to zero at scale $q_{0}$ and evolve according to the generalized DGLAP equations presented below.

For the input used here, and because fermion masses and Yukawa couplings are neglected except for the top quark, several fermion PDFs are identical. The lepton PDFs are independent of generation. Also the right-handed fermion and antifermion PFDs are identical, apart from the top quark, unless they are different at the matching scale $q_{0}$. This is the case only for the up and down quarks. The right-handed top and anti-top are slightly different, since they interact through the Yukawa coupling with $H^{+} H^{-}$, respectively. Thus the number of distinct right-handed quark PDFs is reduced from 12 to 9 , the left-handed leptons from 12 to 4, and the right-handed leptons from 6 to 1, making a total of 36 non-identical PDFs.

\subsection{General evolution equations}

We consider the $x$-weighted PDFs of parton species $i$ at momentum fraction $x$ and scale $q$, $f_{i}(x, q)$. In general they satisfy evolution equations of the following forms:

$$
\begin{aligned}
q \frac{\partial}{\partial q} f_{i}(x, q) & =\sum_{I} \frac{\alpha_{I}(q)}{\pi}\left[P_{i, I}^{V}(q) f_{i}(x, q)+\sum_{j} C_{i j, I} \int_{x}^{z_{\max }^{i j, I}(q)} \mathrm{d} z P_{i j, I}^{R}(z) f_{j}(x / z, q)\right] \\
& \equiv \sum_{I}\left[q \frac{\partial}{\partial q} f_{i}(x, q)\right]_{I} .
\end{aligned}
$$

Here, the sum over $I$ goes over the different interactions in the Standard Model and the notation $\left[q \partial / \partial q f_{i}(x, q)\right]_{I}$ implies that we only keep the terms proportional to the coupling $\alpha_{I}$ when taking the derivative. ${ }^{4}$ For the rest of the section, we will show the evolution of each $f_{i}(x, q)$. We choose $I=1,2,3$ for the pure $\mathrm{U}(1), \mathrm{SU}(2)$ and $\mathrm{SU}(3)$ gauge interactions, $I=Y$ for Yukawa interactions, and $I=M$ for the mixed interaction proportional to

$$
\alpha_{M}(q)=\sqrt{\alpha_{1}(q) \alpha_{2}(q)} .
$$

The first contribution, proportional to $P_{i, I}^{V}$, denotes the virtual contribution to the PDF evolution (the disappearance of a flavor $i$ ), while the second contribution is the real contribution (the appearance of flavor $i$ due to the splitting of a flavor $j$ ). The maximum value of $z$ in the integration of the real contribution depends on the type of splitting and interaction, and we choose

$$
z_{\max }^{i j, I}(q)=\left\{\begin{array}{ll}
1-\frac{m_{V}}{q} & \text { for } I=1,2, \text { and } i, j \notin V \text { or } i, j \in V \\
1 & \text { otherwise }
\end{array},\right.
$$

that is, we apply an infrared cutoff $m_{V}$, of the order of the electroweak scale, when a $B$ or $W$ boson is emitted. This regulates the divergence of the splitting function for those emissions

\footnotetext{
${ }^{4}$ Note that $[\ldots]_{I}$ is only introduced for notational convenience and should not be interpreted as setting all other couplings to zero. In particular, the PDFs appearing on the right-hand side of eq. (2.17) still depend on the value of all coupling constants.
} 


\begin{tabular}{|l|l|}
\hline$\{\mathbf{T}, \mathrm{CP}\}$ & fields \\
\hline$\{0,+\}$ & $2 n_{g} \times q_{R}, n_{g} \times \ell_{R}, n_{g} \times q_{L}, n_{g} \times \ell_{L}, g, W, B, H$ \\
$\{0,-\}$ & $2 n_{g} \times q_{R}, n_{g} \times \ell_{R}, n_{g} \times q_{L}, n_{g} \times \ell_{L}, H$ \\
$\{1,+\}$ & $n_{g} \times q_{L}, n_{g} \times \ell_{L}, B W, H$ \\
$\{1,-\}$ & $n_{g} \times q_{L}, n_{g} \times \ell_{L}, W, H$ \\
$\{2,+\}$ & $W$ \\
\hline
\end{tabular}

Table 1. The $52 \mathrm{PDFs}$ required for the SM evolution can written in a basis with definite conserved quantum numbers. $\left(5 n_{g}+4\right)$ PDFs contribute to the $\{0,+\}$ state, $\left(5 n_{g}+1\right)$ to the $\{0,-\},\left(2 n_{g}+2\right)$ to each to the $\{1,+\}$ and $\{1,-\}$ and 1 to the $\{2,+\}$, where $n_{g}=3$ stands for number of generations.

as $z \rightarrow 1$. Such a cutoff is mandatory for $I=2$ because there are PDF contributions that are $\mathrm{SU}(2)$ non-singlets. The evolution equations for $\mathrm{SU}(3)$ are regular in the absence of a cutoff, as hadron PDFs are color singlets. Similarly for U(1), the unpolarized PDFs have zero hypercharge, ${ }^{5}$ but we include the same cutoff for $I=1$, since the $B$ and $W_{3}$ are mixed in the physical $Z$ and $\gamma$ states.

Note that the precise choice of the cutoff is somewhat arbitrary, and as already mentioned, we choose $m_{V}=100 \mathrm{GeV}$ in this paper. Changing this value changes our results by subleading logarithmic effect, at the same level as other effects not included. However, given that the $\mathrm{SU}(2)$ evolution is double logarithmic, this implies that the ambiguity is single logarithmic for the $\mathrm{SU}(2)$ coupling. By matching our results to fixed order, one would account for these term at first order in $\alpha_{2}$. This is beyond the scope of this paper.

While the flavor basis chosen above is the most intuitive basis, the fact that all 52 PDFs are coupled to one another makes it quite difficult to solve the evolution equations. To decouple some of the equations, it helps to change the basis such that the ingredients have quantum numbers that are conserved in the Standard Model. Choosing the total isospin $\mathbf{T}$ and $\mathrm{CP}$ as the quantum numbers, the PDFs for each set of quantum numbers required are shown in table 1.

Note that in general there can be additional mixed PDFs, which however are zero in our initial conditions and which are not generated in the evolution. In particular, there can be states mixing left-and right-handed fermions, but they are not present in the initial condition when only considering unpolarized beams because those states are not Lorentz scalar. Thus, we can drop these states from our evolution.

The sum of momenta of all non-mixed PDFs in the particle basis is conserved, since it is the momentum of the proton. Momentum conservation applies independently for each interaction since physics would still be coherent if we removed one interaction from the Standard Model.

$$
\sum_{i \neq \mathrm{BW}} \int_{0}^{1} d x\left[q \frac{\partial}{\partial q} f_{i}(x, q)\right]_{I}=0 \text { for } I=1,2,3, Y, M
$$

\footnotetext{
${ }^{5}$ Although there can be contributions with non-zero hypercharge for transversely polarized beams [8].
} 
This is equivalent to the sum over all $\mathbf{T}=0, \mathrm{CP}=+$ PDFs in the isospin and CP basis because only these states contribute to a sum over the PDFs in the particle basis. For the other values of $\mathbf{T}$ and $\mathrm{CP}$, the PDFs correspond to differences of PDFs in the particle basis. For example an isospin $1 \mathrm{PDF}$ is added in PDF of an up-type fermion, but subtracted in the down-type PDF, thus it has no effect on the sum.

Combining eqs. (2.17) and (2.20) gives

$$
\begin{aligned}
0 & =\sum_{i \neq \mathrm{BW}} P_{i, I}^{V} \int_{0}^{1} d x f_{i}(x, q)+\sum_{i, j} C_{i j, I} \int_{0}^{1} \mathrm{~d} x \int_{x}^{z_{\max }^{i j, I}(q)} \mathrm{d} z P_{i j, I}^{R}(z) f_{j}(x / z, q) \\
& =\sum_{i \neq \mathrm{BW}} P_{i, I}^{V} \int_{0}^{1} d x f_{i}(x, q)+\sum_{i, j} C_{i j, I} \int_{0}^{z_{\max }^{i j, I}(q)} \mathrm{d} z P_{i j, I}^{R}(z) \int_{0}^{z} d x f_{j}(x / z, q) \\
& =\sum_{i \neq \mathrm{BW}} P_{i, I}^{V}\left\langle f_{i}(q)\right\rangle+\sum_{i, j} C_{i j, I} \int_{0}^{z_{\max }^{i j, I}(q)} z \mathrm{~d} z P_{i j, I}^{R}(z)\left\langle f_{j}(q)\right\rangle
\end{aligned}
$$

where we have defined the momentum averaged PDF

$$
\left\langle f_{i}(q)\right\rangle \equiv \int_{0}^{1} d x f_{i}(x, q) .
$$

Solving the equation for each of the $\left\langle f_{i}(q)\right\rangle$, since all the input particle PDFs can be set independently, we get

$$
P_{i, I}^{V}(q)=-\sum_{j} C_{j i, I} \int_{0}^{z_{\max }^{j i, I}(q)} z \mathrm{~d} z P_{j i, I}^{R}(z) \text { for } i \neq \mathrm{BW} .
$$

Thus, momentum conservation determines the factor $P_{i, I}^{V}$ for all non-mixed fields in the particle basis.

Note that the result from momentum conservation agrees up to power corrections with the more traditional definition of the virtual corrections as loop insertions on the fields of the PDF, which we denote by $\tilde{P}_{i, I}^{V}$. Summing over possible loops, one has

$$
\begin{aligned}
& \tilde{P}_{f_{i}, I}^{V}(q)=-C_{f f, i, I} \int_{0}^{z_{\max }^{f f, I}(q)} \mathrm{d} z P_{f f, I}^{R}(z) \\
& \tilde{P}_{V_{i}, I}^{V}(q)=-\frac{C_{V V, i, I}}{2} \int_{0}^{z_{\max }^{V V, I}(q)} \mathrm{d} z P_{V V, I}^{R}(z)-\sum_{j \in f, h} C_{j V_{i}, I} \int_{0}^{1} \mathrm{~d} z P_{j V, I}^{R}(z) \\
& \tilde{P}_{H_{i}, I}^{V}(q)=-C_{H H, i, I} \int_{0}^{z_{\max }^{H H, I}(q)} \mathrm{d} z P_{H H, I}^{R}(z)-\sum_{j \in f} C_{j H_{i}, I} \int_{0}^{1} \mathrm{~d} z P_{f H, I}^{R}(z),
\end{aligned}
$$

where $\sum_{j \in f, h}$ is a sum over all fermions and Higgs bosons which are not antiparticles, and

$$
C_{f f, i, I}=\sum_{j} C_{f_{j} f_{i}, I}
$$


and similarly for $C_{V V, i, I}$ and $C_{H H, i, I}$. To see that eqs. (2.23) and (2.24) agree with each other, we will work it out explicitly for the virtual contribution to a fermion. One uses for the fermions that $P_{V f, I}^{R}(z)=P_{f f, I}^{R}(1-z)$ and $C_{f f, I}=C_{V f, I}$ to obtain the correct relation:

$$
\begin{aligned}
P_{f, I}^{V}(q) & =-C_{f f, I}\left[\int_{0}^{z_{\max }} z \mathrm{~d} z P_{f f, I}^{R}(z)+\int_{0}^{1} z \mathrm{~d} z P_{V f, I}^{R}(z)\right] \\
& =-C_{f f, I}\left[\int_{0}^{z_{\max }} z \mathrm{~d} z P_{f f, I}^{R}(z)+\int_{0}^{1}(1-z) \mathrm{d} z P_{f f, I}^{R}(z)\right] \\
& =\tilde{P}_{f, I}^{V}(q)+\ldots,
\end{aligned}
$$

where ... denotes power corrections in $1-z_{\max }$. The argument is exactly the same for $P_{H, I}^{V}(q)$, while for $P_{V, I}^{V}(q)$ one simply uses that $P_{V V, I}^{R}(z)$ and $P_{f V, I}^{R}(z)$, and $P_{h V, I}^{R}(z)$ and $P_{f H, I}^{R}(z)$, are symmetric in $z \leftrightarrow 1-z$ to write $\int z \mathrm{~d} z=\int \mathrm{d} z / 2$. In our implementation of the evolution equations, we use eq. (2.23), to ensure exact momentum conservation without explicit power corrections.

Since the mixed PDF $f_{B W}$ is a pure $\mathbf{T}=1$ state, it does not contribute to the momentum sum. This implies that one cannot derive its associated virtual contribution from momentum conservation. However, using the traditional definition in terms of loops, one sees that in this case the $\mathrm{U}(1)$ and $\mathrm{SU}(2)$ virtual corrections each apply to only one of the two fields involved, and therefore

$$
\tilde{P}_{B W, 1}^{V}(q)=\frac{1}{2} P_{B, 1}^{V}(q), \quad \tilde{P}_{B W, 2}^{V}(q)=\frac{1}{2} P_{W, 2}^{V}(q),
$$

while the virtual contribution is zero for the other interactions.

One can simplify the general evolution equations in eq. (2.17) by defining a full Sudakov factor

$$
\Delta_{i}(q)=\exp \left[\sum_{I} \int_{q_{0}}^{q} \frac{\mathrm{d} q^{\prime}}{q^{\prime}} \frac{\alpha_{I}\left(q^{\prime}\right)}{\pi} P_{i, I}^{V}\left(q^{\prime}\right)\right],
$$

as well as a partial Sudakov factor for each interaction

$$
\Delta_{i, I}(q)=\exp \left[\int_{q_{0}}^{q} \frac{\mathrm{d} q^{\prime}}{q^{\prime}} \frac{\alpha_{I}\left(q^{\prime}\right)}{\pi} P_{i, I}^{V}\left(q^{\prime}\right)\right],
$$

where $q_{0}$ is an arbitrary cutoff, which for convenience we set equal to $m_{V}$. This allows us to write

$$
\left[\Delta_{i, I}(q) q \frac{\partial}{\partial q} \frac{f_{i}(x, q)}{\Delta_{i, I}(q)}\right]_{I}=\frac{\alpha_{I}(q)}{\pi} \sum_{j} C_{i j, I} P_{i j, I}^{R} \otimes f_{j},
$$

where again the notation $[\ldots]_{I}$ implies that only terms from the interaction $I$ are kept. This gives

$$
\begin{aligned}
\Delta_{i}(q) q \frac{\partial}{\partial q}\left[\frac{f_{i}(x, q)}{\Delta_{i}(q)}\right] & =\sum_{I}\left[\Delta_{i, I}(q) q \frac{\partial}{\partial q} \frac{f_{i}(x, q)}{\Delta_{i, I}(q)}\right]_{I} \\
& =\sum_{I} \frac{\alpha_{I}(q)}{\pi} \sum_{j} C_{i j, I} P_{i j, I}^{R} \otimes f_{j},
\end{aligned}
$$

where

$$
P_{i j, I}^{R} \otimes f_{j} \equiv \int_{x}^{z_{\max }^{i j, I}(q)} \mathrm{d} z P_{i j, I}^{R}(z) f_{j}(x / z, q)
$$




\subsection{Splitting functions}

The splitting functions depend only on the type of particles, which for the Standard Model are the spin $1 / 2$ fermions, denoted by $f$, spin 1 gauge bosons, denoted by $V$, as well as spin 0 Higgs bosons, denoted by $H$.

Denoting the three gauge interactions of the Standard Model collectively by $I=G$, the splitting functions involving gauge bosons are given by

$$
\begin{aligned}
P_{f f, G}^{R}(z) & =\frac{1+z^{2}}{1-z}, \\
P_{V f, G}^{R}(z) & =P_{f f, G}(1-z), \\
P_{f V, G}^{R}(z) & =\frac{1}{2}\left[z^{2}+(1-z)^{2}\right], \\
P_{V V, G}^{R}(z) & =2\left[\frac{z}{1-z}+\frac{1-z}{z}+z(1-z)\right] \\
P_{H H, G}^{R}(z) & =\frac{2 z}{1-z}, \\
P_{V H, G}^{R}(z) & =P_{H H, G}^{R}(1-z), \\
P_{H V, G}^{R}(z) & =z(1-z) .
\end{aligned}
$$

The factor of $1 / 2$ in $P_{f V}$ has to be included since we are considering fermions with definite chirality. For the Yukawa interaction $(Y)$, one obtains

$$
\begin{aligned}
P_{f f, Y}^{R}(z) & =\frac{1-z}{2}, \\
P_{H f, Y}^{R}(z) & =P_{f f, Y}^{R}(1-z), \\
P_{f H, Y}^{R}(z) & =\frac{1}{2} .
\end{aligned}
$$

\subsection{Running couplings}

The one-loop running of the gauge couplings $\alpha_{I}(I=1,2,3)$ is given by

$$
\frac{2 \pi}{\alpha_{I}\left(q_{2}\right)}=\frac{2 \pi}{\alpha_{I}\left(q_{1}\right)}+\beta_{I} \ln \frac{q_{2}}{q_{1}},
$$

where, for $n_{g}$ generations and $n_{H}$ Higgs doublets,

$$
\begin{aligned}
& \beta_{1}=-\frac{1}{3} \rho_{1}=-\frac{20}{9} n_{g}-\frac{1}{6} n_{H}=-\frac{41}{6}, \\
& \beta_{2}=\frac{2}{3}\left(11-\rho_{V 2}\right)=\frac{22}{3}-\frac{4}{3} n_{g}-\frac{1}{6} n_{H}=\frac{19}{6}, \\
& \beta_{3}=11-\rho_{3}=11-\frac{4}{3} n_{g}=7 .
\end{aligned}
$$

At scale $M_{Z}=91.2 \mathrm{GeV}$ we take

$$
\sin ^{2} \theta_{W}=\frac{\alpha_{1}}{\alpha_{1}+\alpha_{2}}=0.23, \quad \alpha=\alpha_{2} \sin ^{2} \theta_{W}=\frac{1}{128}, \quad \alpha_{3}=0.118,
$$


which gives

$$
\alpha_{1}\left(M_{Z}\right)=0.0101 \quad \alpha_{2}\left(M_{Z}\right)=0.0340 \quad \alpha_{3}\left(M_{Z}\right)=0.118 .
$$

We set all Yukawa couplings to zero, except for the top Yukawa coupling $\alpha_{Y}=y_{t}^{2} / 4 \pi$. Its running receives significant Yukawa and QCD contributions:

$$
q \frac{\partial \alpha_{Y}}{\partial q}=\frac{\alpha_{Y}}{2 \pi}\left(\beta_{Y} \alpha_{Y}-\beta_{S} \alpha_{3}\right)
$$

where $\beta_{Y}=9 / 2$ and $\beta_{S}=8$. The solution is

$$
\frac{1}{\alpha_{Y}\left(q_{2}\right)}=\frac{\delta}{\alpha_{3}\left(q_{2}\right)}-\left[\frac{\delta}{\alpha_{3}\left(q_{1}\right)}-\frac{1}{\alpha_{Y}\left(q_{1}\right)}\right]\left[\frac{\alpha_{3}\left(q_{1}\right)}{\alpha_{3}\left(q_{2}\right)}\right]^{\gamma},
$$

where

$$
\begin{aligned}
& \gamma=\frac{\beta_{S}}{\beta_{3}}=\frac{24}{33-4 n_{g}}=\frac{8}{7}, \\
& \delta=\frac{\beta_{Y}}{\beta_{S}-\beta_{3}}=\frac{27}{8 n_{g}-18}=\frac{9}{2} .
\end{aligned}
$$

We take $m_{t}\left(m_{t}\right)=163 \mathrm{GeV}$, which implies $\alpha_{Y}\left(m_{t}\right)=0.0349$, and $\alpha_{3}\left(m_{t}\right)=0.109$.

\section{5 $\quad I=3: \mathrm{SU}(3)$ interactions}

We start by considering the well known case of SU(3) interactions. The relevant degrees of freedom are the gluon, as well as left and right-handed quarks. The coupling constants are (with $C_{F}=4 / 3, C_{A}=3, T_{R}=1 / 2$ )

$$
C_{q q, 3}=C_{g q, 3}=C_{F}, \quad C_{q g, 3}=T_{R}, \quad C_{g g, 3}=C_{A} .
$$

This gives for the evolution of a quark or gluon ${ }^{6}$

$$
\begin{aligned}
& {\left[\Delta_{q, 3} q \frac{\partial}{\partial q} \frac{f_{q}}{\Delta_{q, 3}}\right]_{3}=\frac{\alpha_{3}}{\pi}\left[C_{F} P_{f f, G}^{R} \otimes f_{q}+T_{R} P_{f V, G}^{R} \otimes f_{g}\right],} \\
& {\left[\Delta_{g, 3} q \frac{\partial}{\partial q} \frac{f_{g}}{\Delta_{g, 3}}\right]_{3}=\frac{\alpha_{3}}{\pi}\left[C_{A} P_{V V, G}^{R} \otimes f_{g}+\sum_{f} C_{F} P_{V f, G}^{R} \otimes f_{q}\right] .}
\end{aligned}
$$

The Sudakov factor can be obtained from eq. (2.23) using the coupling constants in eq. (2.53). This gives

$$
\begin{aligned}
& P_{q, 3}^{V}(q)=-C_{F} \int_{0}^{1} z \mathrm{~d} z\left[P_{f f, G}^{R}(z)+P_{V f, G}^{R}(z)\right], \\
& P_{g, 3}^{V}(q)=-\int_{0}^{1} z \mathrm{~d} z\left[C_{A} P_{V V, G}^{R}(z)+8 n_{g} T_{R} P_{f V, G}^{R}(z)\right],
\end{aligned}
$$

where we have used in the last line that there are 8 chiral quarks plus antiquarks per generation.

\footnotetext{
${ }^{6}$ From now on we omit the arguments of functions for brevity.
} 
Since the gluon is massless, the upper limit in all the $z$ integrations is equal to 1 [see eq. (2.19)]. This implies that the convolutions $P_{f f, G}^{R} \otimes f_{q}$ and $P_{V V, G}^{R} \otimes f_{g}$ in eqs. (2.54) and (2.55) are both divergent. However, at the same time the virtual splitting functions that enter the Sudakov factors $\Delta_{q, 3}(q)$ and $\Delta_{g, 3}(q)$ defined in eq. (2.29) are also divergent, such that the divergences cancel in the evolution of the actual PDFs. Using +-distributions, as explained in section 3, one obtains evolution equations that are free of any divergences, and which can be implemented numerically. Alternatively, for parton shower implementation, one can impose a cutoff of the form eq. (2.19) with $m_{V}$ replaced by a small parameter $m_{g}>\Lambda_{\mathrm{QCD}}$.

\section{6 $I=1: \mathrm{U}(1)$ interactions}

For $\mathrm{U}(1)$ the relevant degrees of freedom are left- and right-handed fermions (denoted by the subscript $f$ ), as well as the $\mathrm{U}(1)$ gauge boson $B$. The couplings involving fermions and gauge bosons are

$$
C_{f f, 1}=C_{B f, 1}=Y_{f}^{2}, \quad C_{f B, 1}=N_{f} Y_{f}^{2}, \quad C_{B B, 1}=0
$$

where the hypercharges of the different fermions are given by

$$
Y_{q_{L}}=\frac{1}{6}, \quad Y_{u_{R}}=\frac{2}{3}, \quad Y_{d_{R}}=-\frac{1}{3}, \quad Y_{\ell_{L}}=-\frac{1}{2}, \quad Y_{e_{R}}=-1,
$$

and the color factor $N_{f}$ is equal to 3 for quarks and 1 for leptons. The couplings involving the Higgs bosons are

$$
C_{h h, 1}=C_{B h, 1}=C_{h B, 1}=\frac{1}{4},
$$

where $h$ here stands for any of the four Higgs boson PDFs.

Plugging this into the general evolution equation gives

$$
\begin{aligned}
& {\left[\Delta_{f, 1} q \frac{\partial}{\partial q} \frac{f_{f}}{\Delta_{f, 1}}\right]_{1}=\frac{\alpha_{1}}{\pi} Y_{i}^{2}\left[P_{f f, G}^{R} \otimes f_{f}+N_{f} P_{f V, G}^{R} \otimes f_{B}\right],} \\
& {\left[\Delta_{B, 1} q \frac{\partial}{\partial q} \frac{f_{B}}{\Delta_{B, 1}}\right]_{1}=\frac{\alpha_{1}}{\pi}\left[\sum_{f} Y_{f}^{2} P_{V f, G}^{R} \otimes f_{f}+\frac{1}{4} \sum_{h} P_{V H, G}^{R} \otimes f_{h}\right]} \\
& {\left[\Delta_{H, 1} q \frac{\partial}{\partial q} \frac{f_{h}}{\Delta_{H, 1}}\right]_{1}=\frac{\alpha_{1}}{\pi} \frac{1}{4}\left[P_{H H, G}^{R} \otimes f_{h}+P_{H V, G}^{R} \otimes f_{B}\right] .}
\end{aligned}
$$

The virtual splitting functions, required for the Sudakov factor are given by

$$
\begin{aligned}
& P_{f, 1}^{V}(q)=-Y_{f}^{2}\left[\int_{0}^{1-\frac{m_{V}}{q}} z \mathrm{~d} z P_{f f, G}^{R}(z)+\int_{0}^{1} z \mathrm{~d} z P_{V f, G}^{R}(z)\right], \\
& P_{B, 1}^{V}(q)=-n_{g}\left(\frac{11}{9} N_{C}+3\right) \int_{0}^{1} z \mathrm{~d} z P_{f V, G}^{R}(z)-\int_{0}^{1} z \mathrm{~d} z P_{H V, G}^{R}(z), \\
& P_{H, 1}^{V}(q)=-\frac{1}{4}\left[\int_{0}^{1-\frac{m_{V}}{q}} z \mathrm{~d} z P_{H H, G}^{R}(z)+\int_{0}^{1} z \mathrm{~d} z P_{V H, G}^{R}(z)\right],
\end{aligned}
$$


where we have used in the second line that for each generation there are 4 left-handed quarks (one needs to count particles and antiparticles separately), 2 right-handed up-type quarks, 2 right-handed down-type quarks, 4 left-handed leptons and 2 right-handed electrons, and that there are a total of 4 Higgs bosons.

\section{7 $\quad I=2: \mathrm{SU}(2)$ interactions}

The $\mathrm{SU}(2)$ interactions are more complicated, since the emission of $W^{ \pm}$bosons changes the flavor of the emitting particle. This, combined with the $\mathrm{SU}(2)$ breaking in the input hadron PDFs, leads to double-logarithmic scale dependence in the DGLAP evolution, rather than only single-logarithmic dependence as in the evolution based on $\mathrm{U}(1)$ and $\mathrm{SU}(3)$.

The relevant coupling constants are (where $u_{L}$ and $d_{L}$ denote any up- and down-type left-handed fermion)

$$
\begin{aligned}
C_{u_{L} d_{L}, 2}=C_{d_{L} u_{L}, 2}=C_{W^{+} u_{L}, 2}=C_{W^{-} d_{L}, 2} & =\frac{1}{2}, \\
C_{u_{L} u_{L}, 2}=C_{W_{3} u_{L}, 2}=C_{d_{L} d_{L}, 2}=C_{W_{3} d_{L}, 2} & =\frac{1}{4}, \\
C_{u_{L} W^{+}, 2}=C_{d_{L} W^{-}, 2} & =N_{f} \frac{1}{2}, \\
C_{u_{L} W_{3}, 2} & =C_{d_{L} W_{3}, 2}=N_{f} \frac{1}{4}, \\
C_{W^{ \pm} W^{ \pm}, 2}=C_{W^{ \pm} W_{3}, 2} & =C_{W_{3} W^{ \pm}, 2}=1,
\end{aligned}
$$

where as before the color factor $N_{f}=3$ for quarks, 1 for leptons. The couplings of the $W_{3}$ state to the Higgs are given by

$$
C_{h h, 2}=C_{W_{3} h, 2}=C_{h W_{3}, 2}=\frac{1}{4},
$$

where again $h$ stands for any of the 4 Higgs bosons, while those of the charged $W$ states are given by

$$
\begin{aligned}
C_{H^{+} H^{0}, 2} & =C_{H^{0} H^{+}, 2}=C_{H^{+} W^{+}, 2}=C_{W^{+} H^{+}, 2} \\
& =C_{H^{0} W^{-, 2}}=C_{W^{-} H^{0}, 2}=\frac{1}{2} .
\end{aligned}
$$

The couplings for the charge-conjugate states are the same.

This gives for the evolution of the fermions

$$
\begin{aligned}
{\left[\Delta_{f_{L}, 2} q \frac{\partial}{\partial q} \frac{f_{u_{L}}}{\Delta_{f_{L}, 2}}\right]_{2}=\frac{\alpha_{2}}{\pi}\{} & P_{f f, G}^{R} \otimes\left[\frac{f_{d_{L}}}{2}+\frac{f_{u_{L}}}{4}\right] \\
& \left.+N_{f} P_{f V, G} \otimes\left[\frac{f_{W^{+}}}{2}+\frac{f_{W_{3}}}{4}\right]\right\}, \\
{\left[\Delta_{f_{L}, 2} q \frac{\partial}{\partial q} \frac{f_{d_{L}}}{\Delta_{f_{L}, 2}}\right]_{2}=\frac{\alpha_{2}}{\pi}\{} & P_{f f, G}^{R} \otimes\left[\frac{f_{u_{L}}}{2}+\frac{f_{d_{L}}}{4}\right] \\
+ & \left.N_{f} P_{f V, G} \otimes\left[\frac{f_{W^{-}}}{2}+\frac{f_{W_{3}}}{4}\right]\right\} .
\end{aligned}
$$


For the $W^{+}$and $W_{3}$ bosons we have

$$
\begin{aligned}
& {\left[\Delta_{W, 2} q \frac{\partial}{\partial q} \frac{f_{W^{+}}}{\Delta_{W, 2}}\right]_{2}=\frac{\alpha_{2}}{\pi}\{} P_{V V, G}^{R} \otimes\left[f_{W^{+}}+f_{W_{3}}\right]+\frac{1}{2} P_{V H, G}^{R} \otimes\left[f_{H^{+}}+f_{\bar{H}^{0}}\right] \\
&\left.+\sum_{\text {gen }} \frac{1}{2} P_{V f, G} \otimes\left[f_{u_{L}}+f_{\bar{d}_{L}}+f_{\nu_{L}}+f_{\bar{\ell}_{L}}\right]\right\}, \\
& {\left[\Delta_{W, 2} q \frac{\partial}{\partial q} \frac{f_{W_{3}}}{\Delta_{W, 2}}\right]_{2}=\frac{\alpha_{2}}{\pi}\left\{P_{V V, G}^{R} \otimes\left[f_{W^{+}}+f_{W^{-}}\right]+\frac{1}{4} P_{V H, G}^{R} \otimes \sum_{h} f_{h}\right.} \\
&\left.+\frac{1}{4} \sum_{f_{L}} P_{V f, G}^{R} \otimes f_{f_{L}}\right\},
\end{aligned}
$$

where the sum in the last line is over all left-handed fermions and anti-fermions. The equation for the $W^{-}$can be obtained from that of the $W^{+}$by charge conjugation.

Finally, for the Higgs bosons we have

$$
\begin{aligned}
{\left[\Delta_{H, 2} q \frac{\partial}{\partial q} \frac{f_{H^{+}}}{\Delta_{H, 2}}\right]_{2}=\frac{\alpha_{2}}{\pi}\{} & P_{H H, G}^{R} \otimes\left[\frac{f_{H^{0}}}{2}+\frac{f_{H^{+}}}{4}\right] \\
& \left.+P_{H V, G} \otimes\left[\frac{f_{W^{+}}}{2}+\frac{f_{W_{3}}}{4}\right]\right\}, \\
{\left[\Delta_{H, 2} q \frac{\partial}{\partial q} \frac{f_{H^{0}}}{\Delta_{H, 2}}\right]_{2}=\frac{\alpha_{2}}{\pi}\{} & P_{H H, G}^{R} \otimes\left[\frac{f_{H^{+}}}{2}+\frac{f_{H^{0}}}{4}\right] \\
+ & \left.P_{H V, G} \otimes\left[\frac{f_{W^{-}}}{2}+\frac{f_{W_{3}}}{4}\right]\right\} .
\end{aligned}
$$

The virtual splitting functions are

$$
\begin{aligned}
& P_{f, 2}^{V}(q)=-\frac{3}{4}\left[\int_{0}^{1-\frac{m_{V}}{q}} z \mathrm{~d} z P_{f f, G}^{R}(z)+\int_{0}^{1} z \mathrm{~d} z P_{V f, G}^{R}(z)\right] \\
& P_{W, 2}^{V}(q)=-2 \int_{0}^{1-\frac{m_{V}}{q}} z \mathrm{~d} z P_{V V, G}^{R}(z)-n_{g}\left(N_{C}+1\right) \int_{0}^{1} z \mathrm{~d} z P_{f V, G}^{R}(z)-\int_{0}^{1} z \mathrm{~d} z P_{H V, G}^{R}(z) \\
& P_{H, 2}^{V}(q)=-\frac{3}{4}\left[\int_{0}^{1-\frac{m_{V}}{q}} z \mathrm{~d} z P_{H H, G}^{R}(z)+\int_{0}^{1} z \mathrm{~d} z P_{V H, G}^{R}(z)\right]
\end{aligned}
$$

from which the Sudakov factor can be constructed using eq. (2.29).

An important aspect of the $\mathrm{SU}(2)$ evolution equations is that, contrary to the other gauge groups, the dependence on the ratio $m_{V} / q$ does not cancel between the real and virtual splitting functions. As an example, consider the evolution equation for an up-type fermion, given on the first line of eq. (2.74), with the virtual contribution given by the first line of eq. (2.80). The sum of the contributions of real and virtual splitting functions is given by

$$
\frac{\alpha_{2}}{\pi} \int_{0}^{1-\frac{m_{V}}{q}} \mathrm{~d} z \frac{1}{4} P_{f f, G}^{R}(z)\left[2 f_{d_{L}}(x / z)+f_{u_{L}}(x / z)-3 f_{u_{L}}(x)\right]+\ldots
$$


where ... represents less singular terms. Thus, the SU(2) breaking in the proton, which renders $f_{u}(z) \neq f_{d}(z)$, gives rise to a logarithmic dependence on $m_{V} / q$, which leads to a doublelogarithmic dependence upon integration over $q$. As we will see later, the effect of this dependence is to double-logarithmically suppress the $\mathrm{SU}(2)$ breaking effects at high energies.

\section{8 $I=Y$ : Yukawa interactions}

The interaction of Higgs particles with fermions is described by the Yukawa interactions. In this work we only keep the top Yukawa coupling, setting all others to zero. This gives the following couplings

$$
C_{q_{L}^{3} t_{R}, Y}=C_{H^{0} t_{R}, Y}=C_{H^{+} t_{R}, Y}=C_{t_{R} q_{L}^{3}, Y}=C_{\bar{H}^{0} t_{L}, Y}=C_{H^{-} b_{L}, Y}=1,
$$

where $q_{L}^{3}$ denotes either the left-handed top or bottom quark. We furthermore need

$$
C_{t^{R} H^{0}, Y}=C_{t^{R} H^{+}, Y}=C_{t^{L} \bar{H}^{0}, Y}=C_{b_{L} H^{-}, Y}=N_{C} .
$$

This gives contributions to the top quark PDFs, as well as the left-handed bottom PDF:

$$
\begin{aligned}
& {\left[\Delta_{q_{L}^{3}, Y} q \frac{\partial}{\partial q} \frac{f_{t_{L}}}{\Delta_{q_{L}^{3}, Y}}\right]_{Y}=\frac{\alpha_{Y}}{\pi}\left\{P_{f f, Y}^{R} \otimes f_{t_{R}}+N_{C} P_{f H, Y} \otimes f_{\bar{H}^{0}}\right\},} \\
& {\left[\Delta_{t_{R}, Y} q \frac{\partial}{\partial q} \frac{f_{t_{R}}}{\Delta_{t_{R}, Y}}\right]_{Y}=\frac{\alpha_{Y}}{\pi}\left\{P_{f f, Y}^{R} \otimes\left[f_{t_{L}}+f_{b_{L}}\right]+N_{C} P_{f H, Y} \otimes\left[f_{H^{0}}+f_{H^{+}}\right]\right\},} \\
& {\left[\Delta_{q_{L}^{3}, Y} q \frac{\partial}{\partial q} \frac{f_{b_{L}}}{\Delta_{q_{L}^{3}, Y}}\right]_{Y}=\frac{\alpha_{Y}}{\pi}\left\{P_{f f, Y}^{R} \otimes f_{t_{R}}+N_{C} P_{f H, Y} \otimes f_{H^{-}}\right\} .}
\end{aligned}
$$

It also contributes to the evolution of the Higgs bosons:

$$
\begin{gathered}
{\left[\Delta_{H, Y} q \frac{\partial}{\partial q} \frac{f_{H^{+}}}{\Delta_{H, Y}}\right]_{Y}=\frac{\alpha_{Y}}{\pi} P_{H f, Y}^{R} \otimes\left[f_{t_{R}}+f_{\bar{b}_{L}}\right],} \\
{\left[\Delta_{H, Y} q \frac{\partial}{\partial q} \frac{f_{H^{0}}}{\Delta_{H^{0}, Y}}\right]_{Y}=\frac{\alpha_{Y}}{\pi} P_{H f, Y}^{R} \otimes\left[f_{t_{R}}+f_{\bar{t}_{L}}\right] .}
\end{gathered}
$$

The Sudakov factors can be obtained using eq. (2.29) with

$$
\begin{aligned}
P_{q_{L}^{3}, Y}^{V}(q)=\frac{1}{2} P_{t_{R}, Y}^{V}(q) & =-\int_{0}^{1} z \mathrm{~d} z P_{f f, Y}^{R}(z)-\int_{0}^{1} z \mathrm{~d} z P_{H f, Y}^{R}(z), \\
P_{H, Y}^{V}(q) & =-2 N_{C} \int_{0}^{1} z \mathrm{~d} z P_{f H, Y}^{R}(z) .
\end{aligned}
$$

\section{$2.9 \quad I=M:$ mixed $B-W_{3}$ interactions}

Finally, we need to consider the evolution involving the mixed $B W$ boson PDF. The nonvanishing couplings are

$$
\begin{aligned}
C_{B W f_{u}, M} & =-C_{B W f_{d}, M}=2 \frac{Y_{f}}{2}, \\
C_{f_{u} B W, M} & =-C_{f_{d} B W, M}=N_{f} \frac{Y_{f}}{2},
\end{aligned}
$$


where $f_{u}$ and $f_{d}$ represent the up- and down-type left-handed fermions and anti-fermions of all generations. Since $Y_{\bar{f}}=-Y_{f}$ and $T_{3 \bar{f}}=-T_{3 f}$, the couplings for fermions and antifermions are identical. The factor of 2 in the first line comes from our definition of $f_{B W}$ as the sum of $B W$ and $W B$ contributions. The diagonal coefficients $C_{f_{u} f_{u}, M}$ and $C_{f_{d} f_{d}, M}$ are zero because there is no vector boson with both $\mathrm{U}(1)$ and $\mathrm{SU}(2)$ interactions. For the same reason, there are no Sudakov factors associated with the mixed interaction. The couplings involving the Higgs bosons are

$$
\begin{aligned}
C_{B W H^{+}, M} & =-C_{B W H^{0}, M}=\frac{1}{2}, \\
C_{H^{+} B W, M} & =-C_{H^{0} B W, M}=\frac{1}{4},
\end{aligned}
$$

where, as for the fermions, the same relations hold for the charge-conjugate states.

Plugging these into the general evolution equation gives

$$
\begin{aligned}
& {\left[q \frac{\partial}{\partial q} f_{f_{u}}\right]_{M}=\frac{\alpha_{M}}{\pi} \frac{Y_{f}}{2} N_{f} P_{f V, G}^{R} \otimes f_{B W},} \\
& {\left[q \frac{\partial}{\partial q} f_{f_{d}}\right]_{M}=-\frac{\alpha_{M}}{\pi} \frac{Y_{f}}{2} N_{f} P_{f V, G}^{R} \otimes f_{B W}} \\
& {\left[q \frac{\partial}{\partial q} f_{B W}\right]_{M}=\frac{\alpha_{M}}{\pi}\left[\sum_{f_{u}} Y_{f} P_{V f, G}^{R} \otimes f_{f_{u}}-\sum_{f_{d}} Y_{f} P_{V f, G}^{R} \otimes f_{f_{d}}\right.} \\
& \left.+\frac{1}{2} \sum_{h_{u}} P_{V H, G}^{R} \otimes f_{h_{u}}-\frac{1}{2} \sum_{h_{d}} P_{V H, G}^{R} \otimes f_{h_{d}}\right], \\
& {\left[q \frac{\partial}{\partial q} f_{h_{u}}\right]_{M}=\frac{\alpha_{M}}{\pi} \frac{1}{4} P_{H V, G}^{R} \otimes f_{B W},} \\
& {\left[q \frac{\partial}{\partial q} f_{h_{d}}\right]_{M}=-\frac{\alpha_{M}}{\pi} \frac{1}{4} P_{H V, G}^{R} \otimes f_{B W} .}
\end{aligned}
$$

As already discussed, the mixed gauge field PDF $f_{B W}$ has Sudakov factors associated with the $\mathrm{U}(1)$ and $\mathrm{SU}(2)$ interactions, given by eq. (2.29). Since there is no corresponding real emission term in the evolution equation for $f_{B W}$, it evolves double-logarithmically and is suppressed at high scales relative to the unmixed PDFs.

\section{Implementation details}

Our treatment assumes that the SM PDFs at very high energies can be obtained by smoothly matching the broken and unbroken symmetry regimes at a matching scale $q_{0} \sim$ $m_{V}$, which in practice we take to be $100 \mathrm{GeV}$. Our input PDFs at $100 \mathrm{GeV}$ are obtained as follows. We take the CT14qed PDF set [15] at $10 \mathrm{GeV}$ and replace the photon PDF by that of the LUXqed set [16]. We do not use the CT14qed photon because the LUXqed photon, while being consistent with CT14qed, has much smaller uncertanties and a smoother $x$ dependence. The LUXqed PDF set combines the PDF4LHC15_nnlo_100 parton set [31] with a determination of the photon PDF from structure function and elastic form factor 
fits in electron-proton scattering. However, we do not use the LUXqed partons, because being NNLO they are not positive-definite, which we require for our LO treatment and is satisfied by CT14qed.

We evolve this hybrid CT14-LUX PDF set from 10 to $100 \mathrm{GeV}$ using leading-order QCD plus QED evolution, which incidentally generates the charged leptons. The resulting parton, photon and lepton PDFs form our input to the unbroken SM evolution upwards from $100 \mathrm{GeV}$. The input left- and right-handed fermion PDFs are identical. The input $W^{3}, B$ and mixed $B / W^{3}$ PDFs are determined by the photon (and the absence of the $Z^{0}$ ) at the matching scale according to eq. (2.7). The remaining vector boson, neutrino and Higgs PDFs are all generated dynamically starting from zero at the matching scale.

The equations given in sections 2.5 to 2.9 completely define the evolution of all parton distribution functions in the unbroken symmetry regime. However, as already explained, one can rewrite the equations slightly to make them more amenable to a numerical implementation. First, switching to a basis of states with well-defined isospin decouples the set of 52 equations to some degree. In this new basis another transformation eliminates the double logarithmic sensitivity to the ratio $m_{V} / q$. Second, by combining the virtual and real splitting functions into +-distributions, one can reduce numerical sensitivity to the cutoff of the $z$ integrations. We will now discuss these simplifications in turn.

\subsection{Switching to a basis of conserved quantum numbers}

As we already explained in section 2.2, the set of 52 evolution equations can be decoupled to some degree by switching to a basis of well-defined isospin $\mathbf{T}$ and $\mathrm{CP}$. Writing a fermion PDF with $\mathbf{T}$ and $\mathrm{CP}$ as $f_{i}^{\mathbf{T C P}}$, we write the left-handed fermions as

$$
\begin{array}{lll}
f_{f_{L}}^{0+}=\frac{1}{4}\left(f_{u_{L}}+f_{d_{L}}+f_{\bar{d}_{L}}+f_{\bar{u}_{L}}\right), & f_{f_{L}}^{1+}=\frac{1}{4}\left(f_{u_{L}}-f_{d_{L}}-f_{\bar{d}_{L}}+f_{\bar{u}_{L}}\right), \\
f_{f_{L}}^{0-}=\frac{1}{4}\left(f_{u_{L}}+f_{d_{L}}-f_{\bar{d}_{L}}-f_{\bar{u}_{L}}\right), & f_{f_{L}}^{1-}=\frac{1}{4}\left(f_{u_{L}}-f_{d_{L}}+f_{\bar{d}_{L}}-f_{\bar{u}_{L}}\right),
\end{array}
$$

where $u_{L}$ and $d_{L}$ refer to left-handed up- and down-type fermions. Right-handed fermions are given by

$$
f_{f_{R}}^{0+}=\frac{1}{2}\left(f_{f_{R}}+f_{\bar{f}_{R}}\right), \quad f_{f_{R}}^{0-}=\frac{1}{2}\left(f_{f_{R}}-f_{\bar{f}_{R}}\right) .
$$

The $\mathrm{SU}(3)$ and $\mathrm{U}(1)$ boson PDFs have $\mathbf{T}=0, \mathrm{CP}=+$

$$
f_{g}^{0+}=f_{g}, \quad f_{B}^{0+}=f_{B},
$$

while the $\mathrm{SU}(2)$ boson PDFs can have $\mathbf{T}=0,1,2$ with respectively $\mathrm{CP}=+,-,+$

$$
\begin{aligned}
f_{W}^{0+} & =\frac{1}{3}\left(f_{W^{+}}+f_{W^{-}}+f_{W^{0}}\right), \quad f_{W}^{1-}=\frac{1}{2}\left(f_{W^{+}}-f_{W^{-}}\right), \\
f_{W}^{2+} & =\frac{1}{6}\left(f_{W^{+}}+f_{W^{-}}-2 f_{W^{0}}\right) .
\end{aligned}
$$

The mixed $B W$ boson state is a combination of $0^{-}$and $1^{-}$and therefore its PDF has $\mathbf{T}=1, \mathrm{CP}=+$

$$
f_{B W}^{1+}=f_{B W}
$$


For the Higgs boson, one writes similarly to the fermions

$$
\begin{array}{ll}
f_{H}^{0+}=\frac{1}{4}\left(f_{H^{+}}+f_{H^{0}}+f_{\bar{H}^{0}}+f_{H^{-}}\right), & f_{H}^{1+}=\frac{1}{4}\left(f_{H^{+}}-f_{H^{0}}-f_{\bar{H}^{0}}+f_{H^{-}}\right), \\
f_{H}^{0-}=\frac{1}{4}\left(f_{H^{+}}+f_{H^{0}}-f_{\bar{H}^{0}}-f_{H^{-}}\right), & f_{H}^{1+}=\frac{1}{4}\left(f_{H^{+}}-f_{H^{0}}+f_{\bar{H}^{0}}-f_{H^{-}}\right) .
\end{array}
$$

In terms of these states the longitudinal vector boson and Higgs PDFs are then, using eq. (2.16),

$$
\begin{aligned}
& f_{W_{L}^{+}}=f_{H}^{0+}+f_{H}^{1+}+f_{H}^{0-}+f_{H}^{1-}, \\
& f_{W_{L}^{-}}=f_{H}^{0+}+f_{H}^{1+}-f_{H}^{0-}-f_{H}^{1-}, \\
& f_{Z_{L}}=f_{h}=f_{H}^{0+}-f_{H}^{1+} .
\end{aligned}
$$

\subsection{Cancellation of double-logarithmic dependence in evolution equations}

In the $\{\mathbf{T}, \mathrm{CP}\}$ basis the singular contributions to the evolution equations (those that are proportional to the splitting functions $P_{f f, G}^{R}(z), P_{V V, G}^{R}(z)$ and $P_{H H, G}^{R}(z)$, which diverge in the limit $z \rightarrow 1$ ) are diagonal,

$$
\left[\Delta_{i, I} q \frac{\partial}{\partial q} \frac{f_{i}^{\mathbf{T C P}}}{\Delta_{i, I}}\right]_{I}=\frac{\alpha_{I}}{\pi} D_{i, I}^{\mathbf{T C P}} P_{i i, I}^{R} \otimes f_{i}^{\mathbf{T C P}}+\ldots,
$$

such that the PDF multiplying the divergent splitting function is the same as that appearing on the left-hand side. Here, as in $f_{i}^{\mathbf{T} C P}$, the label $i$ now refers to a parton species $f, V, H$ rather than a particular parton. Recalling that the Sudakov factor takes the form

$$
\begin{aligned}
\Delta_{i, I}(q) & =\exp \left[\int_{q_{0}}^{q} \frac{\mathrm{d} q^{\prime}}{q^{\prime}} \frac{\alpha_{I}\left(q^{\prime}\right)}{\pi} P_{i, I}^{V}\left(q^{\prime}\right)\right] \\
& =\exp \left[-C_{i, I} \int_{q_{0}}^{q} \frac{\mathrm{d} q^{\prime}}{q^{\prime}} \frac{\alpha_{I}\left(q^{\prime}\right)}{\pi} \int_{0}^{z_{\max }^{i i, I}(q)} z \mathrm{~d} z P_{i i, I}^{R}(z)+\ldots\right],
\end{aligned}
$$

where ... represents less divergent terms, and

$$
C_{i, I}=\sum_{k \in i} C_{k l, I} \text { for } l \in i,
$$

where $k$ and $l$ are particular partons, we have

$$
\begin{aligned}
{\left[q \frac{\partial}{\partial q} f_{i}^{\mathbf{T C P}}\right]_{I} } & =\frac{\alpha_{I}}{\pi}\left[D_{i, I}^{\mathbf{T C P}} P_{i i, I}^{R} \otimes f_{i}^{\mathbf{T C P}}+P_{i, I}^{V} f_{i}^{\mathbf{T C P}}\right]+\ldots, \\
& =\frac{\alpha_{I}}{\pi}\left[D_{i, I}^{\mathbf{T C P}} P_{i i, I}^{+} \otimes f_{i}^{\mathbf{T C P}}+\left(1-\frac{D_{i, I}^{\mathbf{T C P}}}{C_{i, I}}\right) P_{i, I}^{V} f_{i}^{\mathbf{T C P}}\right]+\ldots,
\end{aligned}
$$

where

$$
\begin{aligned}
P_{i i, I}^{+} \otimes f_{i} & \equiv P_{i i, I}^{R} \otimes f_{i}+\frac{P_{i, I}^{V}}{C_{i, I}} f_{i} \\
& =\int_{0}^{z_{\max }^{i i, I}(q)} \mathrm{d} z\left[P_{i i, I}^{R}(z) \theta(z>x) f(x / z, q)-z P_{i i, I}^{R}(z) f(x, q)\right]+\ldots
\end{aligned}
$$


The +-prescription defined by eq. (3.17) regulates the divergence in the integrand as $z \rightarrow 1$ and therefore if we define the modifying factor

$$
\begin{aligned}
F_{i, I}^{\mathbf{T C P}}(q) & =\exp \left[\left(1-\frac{D_{i, I}^{\mathbf{T C P}}}{C_{i, I}}\right) \int_{q_{0}}^{q} \frac{\mathrm{d} q^{\prime}}{q^{\prime}} \frac{\alpha_{I}\left(q^{\prime}\right)}{\pi} P_{i, I}^{V}\left(q^{\prime}\right)\right] \\
& =\left[\Delta_{i, I}(q)\right]^{1-D_{i, I}^{\mathbf{T C P}} / C_{i, I}},
\end{aligned}
$$

then the evolution equation (3.13) becomes

$$
\left[F_{i, I}^{\mathbf{T C P}} q \frac{\partial}{\partial q} \frac{f_{i}^{\mathbf{T C P}}}{F_{i, I}^{\mathbf{T C P}}}\right]_{I}=\frac{\alpha_{I}}{\pi} D_{i, I}^{\mathbf{T C P}} P_{i i, I}^{+} \otimes f_{i}^{\mathbf{T C P}}+\ldots,
$$

with no logarithmic dependence on $m_{V} / q$ on the right-hand side.

For all interactions except $\mathrm{SU}(2)$, one can show that $D_{i, I}^{\mathrm{TCP}}=C_{i, I}$, so that the modifying factor (3.18) is unity. ${ }^{7}$ For $\mathrm{SU}(2)$ we have explicitly: ${ }^{8}$

$$
C_{f, 2}=C_{H, 2}=\frac{3}{4}, \quad C_{V, 2}=2,
$$

while

$$
\begin{aligned}
& D_{f, 2}^{0 \pm}=D_{H, 2}^{0 \pm}=\frac{3}{4}, \quad D_{f, 2}^{1 \pm}=D_{H, 2}^{1 \pm}=-\frac{1}{4}, \\
& D_{V, 2}^{0+}=2, \quad D_{V, 2}^{1-}=1, \quad D_{V, 2}^{2+}=-1,
\end{aligned}
$$

so that

$$
\begin{aligned}
F_{f, 2}^{0 \pm} & =F_{H, 2}^{0 \pm}=1, & F_{f / H, 2}^{1 \pm} & =\Delta_{f / H, 2}^{4 / 3}, \\
F_{V, 2}^{0+} & =1, \quad F_{V, 2}^{1-}=\Delta_{V, 2}^{1 / 2}, & F_{V, 2}^{2+} & =\Delta_{V, 2}^{3 / 2} .
\end{aligned}
$$

For the mixed PDF $f_{B W}$ we have $D_{B W, 2}^{1+}=0$ and therefore

$$
F_{B W, 2}^{1+}=\Delta_{B W, 2}=\Delta_{V, 2}^{1 / 2}=F_{V, 2}^{1-}
$$

The equations finally used to evolve the PDFs in the conserved-quantum-number basis are given in appendix $\mathrm{A}$.

\section{Results}

We begin by showing how the PDFs of strongly interacting particles are changed by including the evolution of the full Standard Model. Figure 1 shows results on the evolution of left- and right-handed quark PDFs, shown solid and dashed respectively, normalized to their values assuming pure QCD evolution. In each plot we show the results at three different scales, namely $q=10^{4} \mathrm{GeV}, q=10^{6} \mathrm{GeV}$ and $q=10^{8} \mathrm{GeV}$. The values of $10^{6}$ and $10^{8} \mathrm{GeV}$ are of course far away from energy scales one can reach at any collider in the near or distant future. However, showing the results at such unattainable values helps to illustrate their approach to asymptotic behavior.

\footnotetext{
${ }^{7}$ For the $\mathrm{U}(1)$ interaction one has $D_{i, 1}^{\mathbf{T C P}}=C_{i, 1}=0$, and we choose to set the modifying factor to 1 in this case.

${ }^{8}$ Here we have used the numerical values for the Casimir operator eigenvalues for the corresponding $\mathrm{SU}(2)$ representations, $C_{F}^{\mathrm{SU}(2)}=3 / 4, C_{A}^{\mathrm{SU}(2)}=2$.
} 

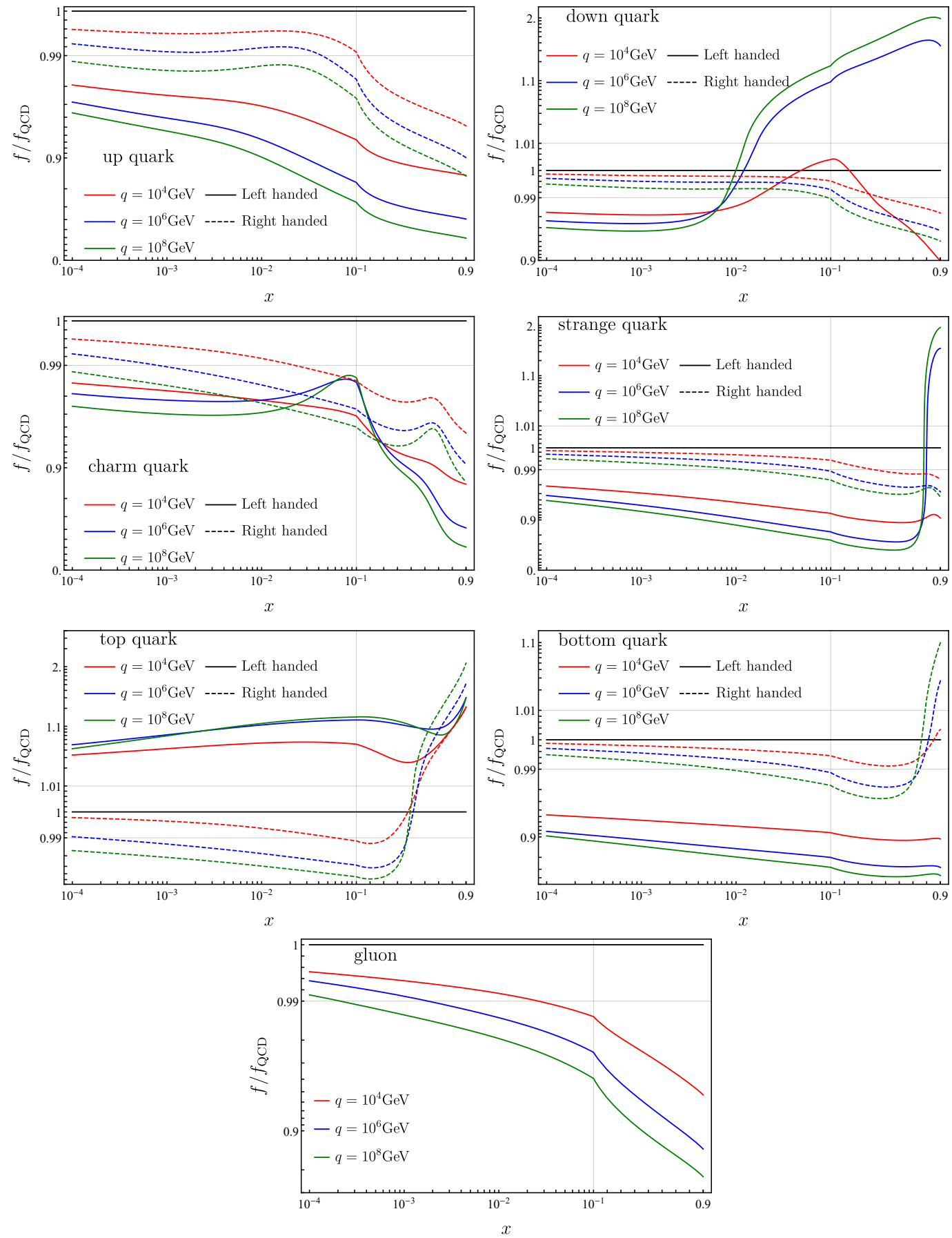

Figure 1. Quark and gluon PDFs in the full unbroken SM, divided by their values assuming pure QCD evolution only. Left- and right-handed quark chiralities are solid and dashed, respectively. The thin gray lines show where the scales on the $\mathrm{x}$ - and/or $\mathrm{y}$-axes switch between linear and logarithmic.

All the light quarks (and antiquarks, not shown) evolve to lower values compared to pure QCD at small $x$, due to an overall loss of energy to the electroweak gauge bosons through the additional splittings $q \rightarrow q W$ and $q \rightarrow q B$. At higher $x$ values, the up and 
down quarks (top row) exhibit different behaviors, with the left-handed up PDF evolving more rapidly to lower values compared to pure QCD, while the down quark eventually evolves to higher values. This is because the left-handed up and down distributions evolve towards each other, their difference being double-logarithmically suppressed at high scales. The right-handed quark PDFs have no double-logarithmic component and evolve to slightly lower values than pure QCD, due to energy loss through the additional splitting $q_{R} \rightarrow q_{R} B$.

The asymmetry between left-handed charm and strange quarks also evolves doublelogarithmically towards zero, primarily through a more rapid decrease of the strange PDF. At high $x$ the behavior is more complicated because the input CT14qed charm PDF is larger than the strange above $x \sim 0.7$. The right-handed quarks behave qualitatively the same as those of the first generation.

The left-handed top and bottom quarks also must evolve towards equal values, which in this case means that the top has higher values than in pure QCD, while the bottom evolution looks similar to strange, relative to pure QCD. The right-handed $b$-quark behaves qualitatively like the right-handed quarks of the first and second generation, while the righthanded top quark, being generated purely dynamically, behaves differently at large $x$. Since the right-handed top has vanishing initial condition, the splitting $t_{R} \rightarrow t_{R} B$, which would decrease the PDF, is sub-dominant compared to the process $B \rightarrow t_{R} \bar{t}_{R}$. This means that at large $x$ the right-handed top PDF is increased, rather than decreased.

The effect on the gluon PDF is shown in last row of figure 1. While the effects are quite small up to $q \sim 10^{4} \mathrm{GeV}$, at larger scales the back-reaction from the changing quark PDFs is affecting the gluon PDF at an appreciable level.

It is interesting to study how rapidly electroweak symmetry is restored. To illustrate this, we show the asymmetry

$$
A^{q_{L}}=\frac{f_{u_{L}}-f_{d_{L}}}{f_{u_{L}}+f_{d_{L}}}
$$

compared to the result if only QCD evolution were turned on. This asymmetry ratio is shown in figure 2 for the three generation of quarks as a function of $q$, for various values of $x$. For all generations the asymmetry decreases as $q$ gets larger, driving the PDFs of the different isospin states towards each other. The onset of the deviation from pure QCD is in the range $1-10 \mathrm{TeV}$. The ratio between the full asymmetry and the result using only QCD evolution is given by

$$
A^{q_{L}}(x, q) \sim\left[\Delta_{f, 2}(q)\right]^{4 / 3} A_{\mathrm{QCD}}^{q_{L}}(x, q)
$$

where $\Delta_{f, 2}(q)$ is the fermion Sudakov factor, as given in eq. (3.18), independent of the generation.

Next, we study the size of the PDFs of particles not charged under the strong interaction. Since these PDFs are only generated by emissions due to the U(1), SU(2) or Yukawa interactions, they are vanishing at all scales if one is including only $\mathrm{SU}(3)$ evolution. The only exception is the photon, which has a non-vanishing initial condition at $q=100 \mathrm{GeV}$. Figure 3 shows results on the electroweak boson PDFs normalized to the gluon PDF, both evolved using the full Standard Model. One can see that the electroweak gauge boson PDFs become a significant fraction of the gluon PDF, especially at large values of $x$. The photon PDF is the largest mainly because it has a non-zero input. The PDF for the $W^{+}$ 


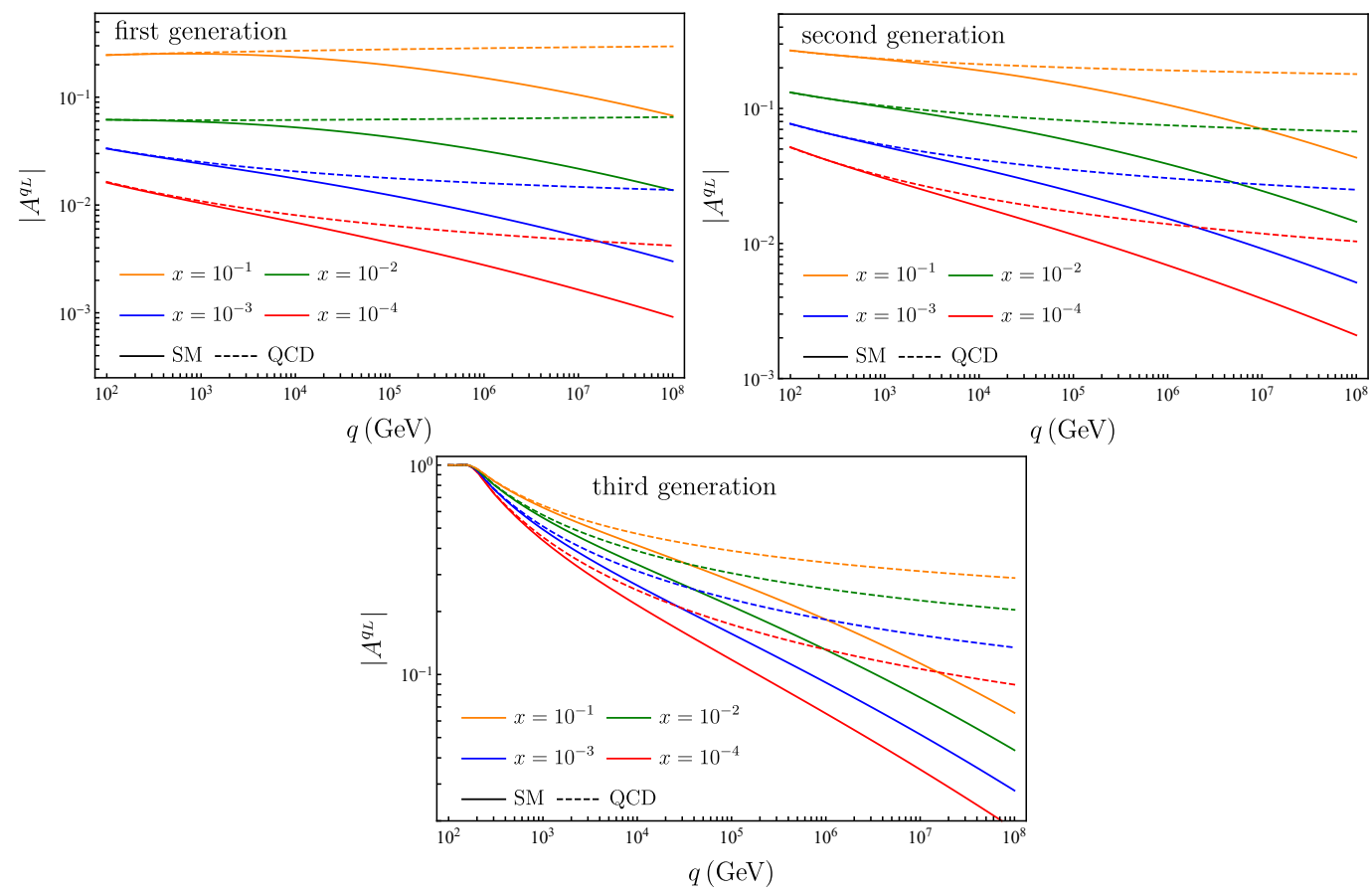

Figure 2. Asymmetry between up-isospin and down-isospin left-handed quark PDFs, defined in eq. (4.1), in the full unbroken SM, compared to the result when only QCD evolution is included.

boson is initially larger than the $W^{-}$boson PDF at large $x$ because the $W^{+}$is mainly generated through emissions from the up-quark, whose PDF is larger than the down-quark which mainly generates the $W^{-}$. Since the difference between $W^{+}$and $W^{-}$has isospin 1 , the $W^{+}$evolves more slowly and the $W^{-}$more rapidly, so that they approach each other at high $q$. At low $x$ they are more similar as are the up-quark and down-quark PDFs. The $Z^{0} \mathrm{PDF}$ is similar to the $W^{+}$but it is smaller at low $x$ and larger at large $x$. The mixed $\gamma Z$ PDF is small and positive at small $x$ and negative at large $x$. There is no constraint to be positive definite for a mixed PDF as it is the product of two amplitudes rather than the square modulus of one. Its absolute value becomes very large at large $x$ and $q$.

We also show the PDFs for the longitudinally polarized gauge bosons, the Higgs boson, the mixed PDF between the Higgs and the $Z_{L}$ and the leptons. The $Z_{L} \mathrm{PDF}$ is the same as the Higgs in our approximation, see eq. (2.16), so we do not make a separate plot for it. The boson PDFs are shown in figure 4, and the leptons in figure 5, both normalized to the gluon. Both are expected to be much smaller than the transverse vector boson PDFs, because they are generated via a second order effect of emission from the vector bosons and via Yukawa emission from the top and bottom quarks, which are much smaller than the up and down quarks. The mixed PDF is even smaller because it is generated by the asymmetry between transverse $W^{+}$and $W^{-}$PDFs and the top and anti-top PDFs. The $W_{L}^{+}$and $W_{L}^{-}$PDFs are very similar, for the same reason.

As a final result, we study several parton luminosities, choosing a future $100 \mathrm{TeV} p p$ collider as a reference. While the energy scales that can be reached at such a collider 

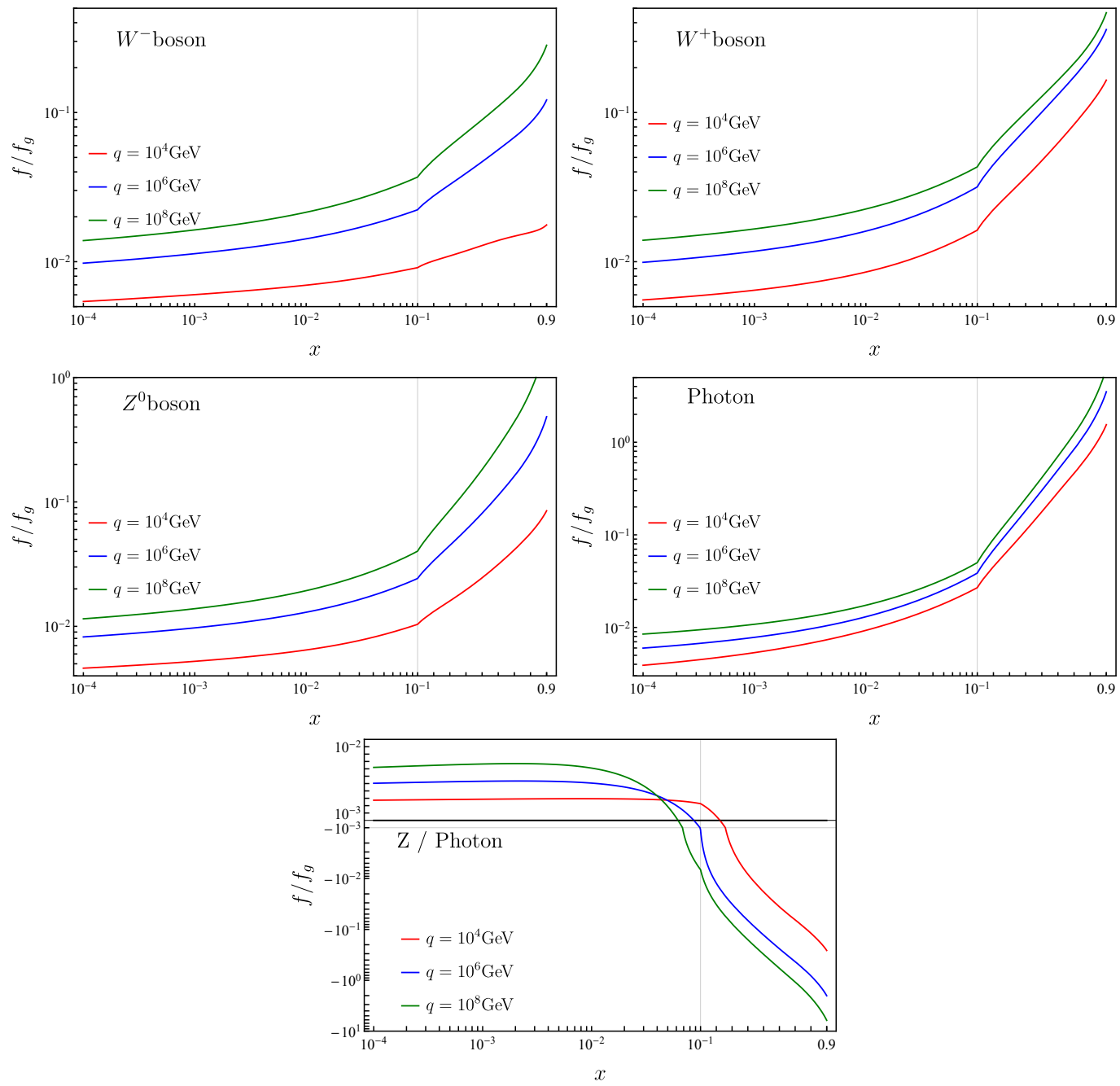

Figure 3. Electroweak bosons PDF normalized by the gluon PDF. The thin gray lines show where the scales on the $\mathrm{x}$ - and/or $\mathrm{y}$-axes switch between linear and logarithmic.

are not quite large enough to get $\mathcal{O}(1)$ effects, the effects of the full Standard Model evolution are still numerically relevant. In figure 6 we show the $q_{L} \bar{q}_{L}$ luminosities for the six different quark flavors, normalized to their values if only QCD evolution is taken into account. One can see that all except the $t \bar{t}$ luminosity are reduced appreciably from their values if only QCD evolution were taken into account. This will affect searches for $Z^{\prime}$-like particles at a future $100 \mathrm{TeV}$ collider. The $d \bar{d}$ luminosity is decreasing more slowly as the double-logarithmic evolution drives it larger than QCD at high $x$ (see figure 1).

We also show selected luminosities of vector bosons combined with quarks, normalized to the average of the $u \bar{u}$ and $d \bar{d}$ luminosities. One can see that luminosities involving one transverse vector boson become of comparable magnitude to the $q \bar{q}$ luminosities. Luminosities involving the longitudinal gauge and Higgs bosons are much smaller.

Finally, to illustrate the uncertainties associated with subleading terms, we show in tables 2 and 3 the dependence of some integrated PDFs (momentum fractions) on the in- 

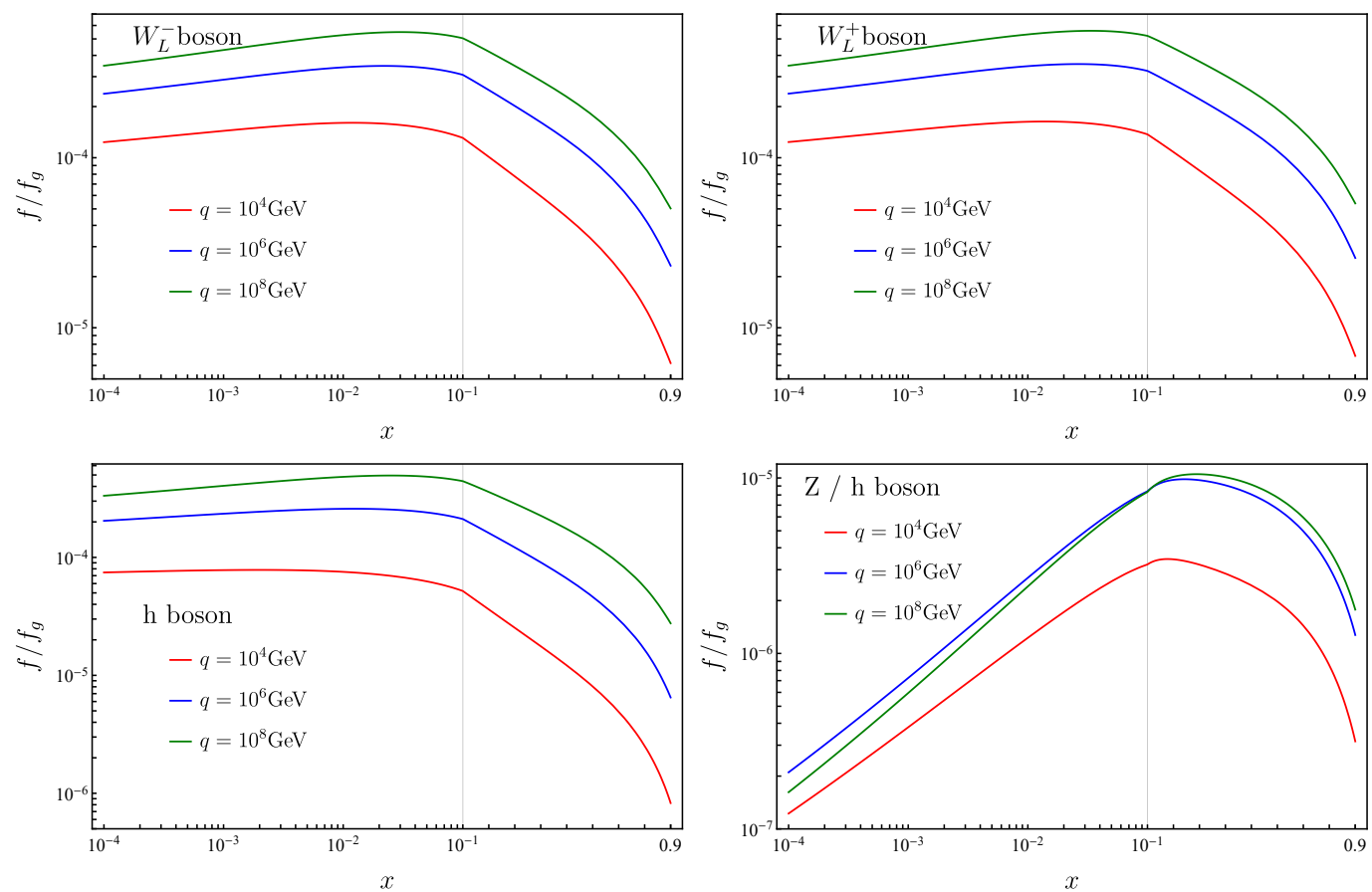

Figure 4. Longitudinal gauge and Higgs bosons PDFs normalized by the gluon PDF. The $Z_{L}$ $\mathrm{PDF}$ is the same as the $h \mathrm{PDF}$. The $h Z_{L} \mathrm{PDF}$ is purely imaginary and we show the result divided by $i$. The thin gray line shows where the scales on the $\mathrm{x}$ - and/or $\mathrm{y}$-axes switch between linear and logarithmic.
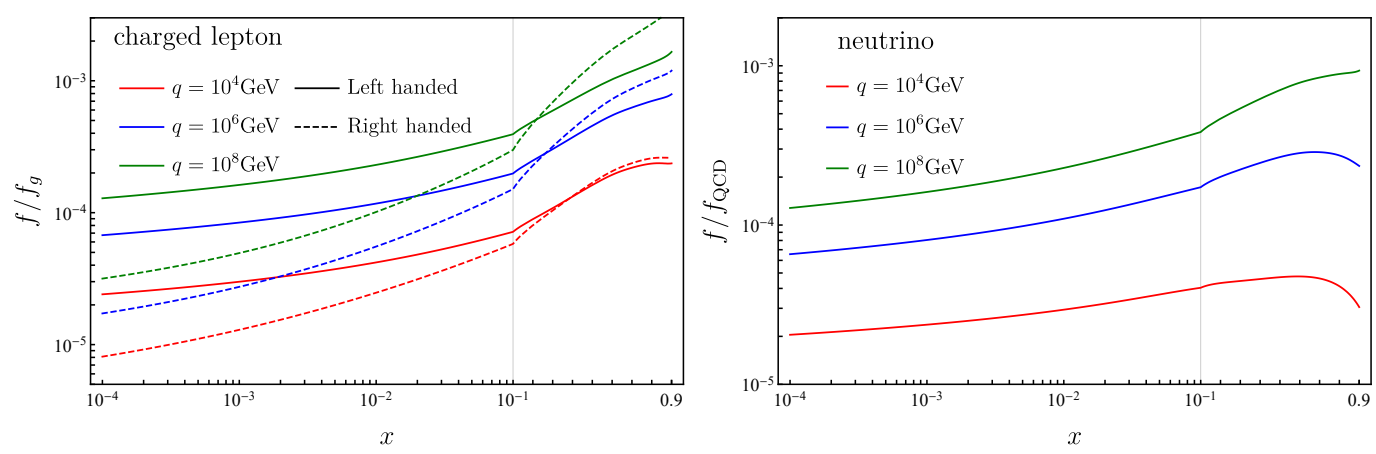

Figure 5. First generation lepton PDFs normalized by the gluon PDF. Since we treat leptons as massless, and all leptons have the same initial condition, the results for the other 2 generations are identical. The thin gray line shows where the scales on the $\mathrm{x}$ - and/or $\mathrm{y}$-axes switch between linear and logarithmic.

frared cutoff $m_{V}$ and matching scale $q_{0}$. We see that there are variations in the electroweak PDFs of the order of $\pm 10 \%$ at $10 \mathrm{TeV}$ and $5 \%$ at $100 \mathrm{TeV}$ for the ranges of parameters indicated. The relative variations in the light quark PDFs are smaller as they are dominated by QCD evolution. There are of course in addition the usual uncertainties associated with the input PDFs and higher-order QCD corrections. 

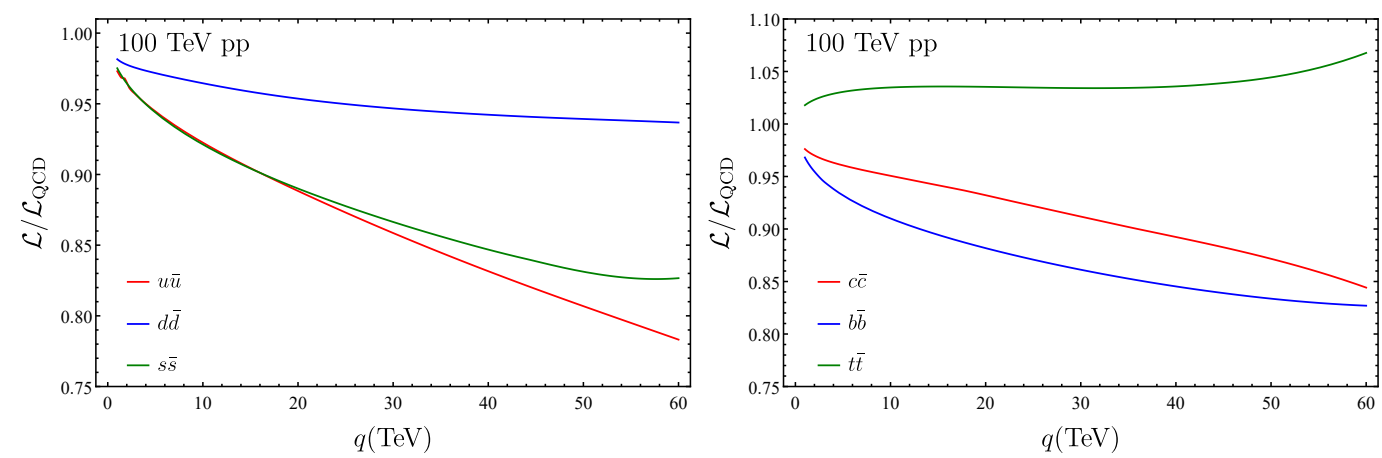

Figure 6. Quark anti-quark luminosity in the full unbroken SM, divided by their values assuming pure QCD evolution only.
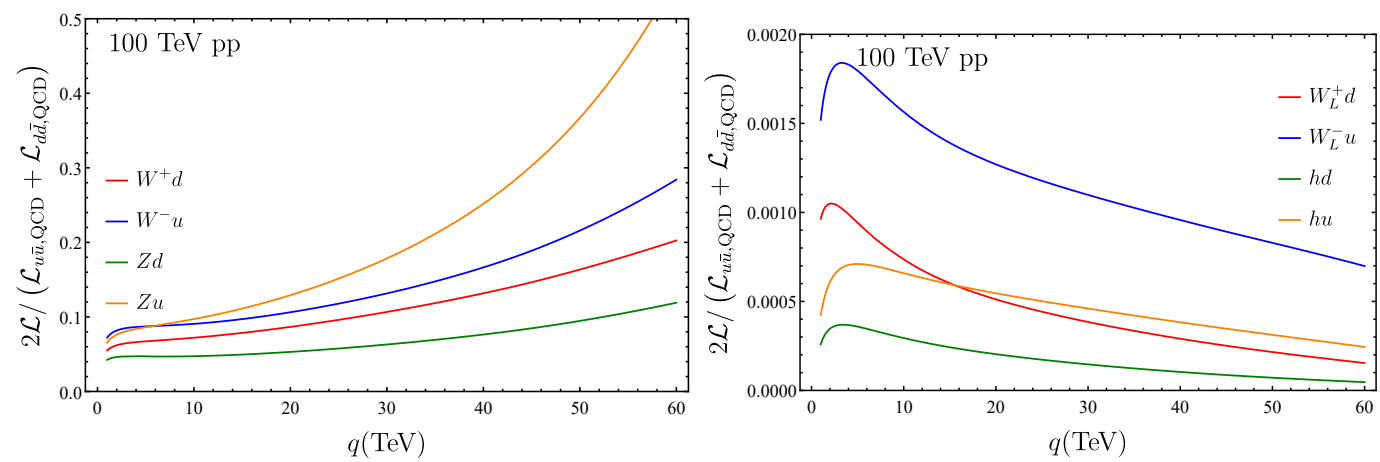

Figure 7. $V q$ and $H q$ luminosity in the full unbroken SM, divided by the average of $u \bar{u}$ and $d \bar{d}$ luminosity assuming pure QCD evolution only.

\begin{tabular}{|c|c|c|c|c|c|c|c|c|}
\hline$m_{V} / \mathrm{GeV}$ & $q_{0} / \mathrm{GeV}$ & $u_{L}$ & $t_{L}$ & $W_{T}^{+}$ & $W_{T}^{-}$ & $e_{L}^{-}$ & $\nu_{e}$ & $h$ \\
\hline 100 & 100 & 8.51 & 0.43 & 0.46 & 0.33 & 0.0019 & 0.0012 & 0.0026 \\
50 & 100 & 8.42 & 0.43 & 0.46 & 0.34 & 0.0019 & 0.0012 & 0.0027 \\
50 & 200 & 8.48 & 0.44 & 0.39 & 0.29 & 0.0017 & 0.0009 & 0.0025 \\
100 & 200 & 8.56 & 0.43 & 0.39 & 0.29 & 0.0017 & 0.0009 & 0.0024 \\
200 & 200 & 8.64 & 0.42 & 0.39 & 0.28 & 0.0017 & 0.0009 & 0.0024 \\
\hline
\end{tabular}

Table 2. Momentum fractions (\%) carried by various parton species at scale $q=10 \mathrm{TeV}$.

\begin{tabular}{|c|c|c|c|c|c|c|c|c|}
\hline$m_{V} / \mathrm{GeV}$ & $q_{0} / \mathrm{GeV}$ & $u_{L}$ & $t_{L}$ & $W_{T}^{+}$ & $W_{T}^{-}$ & $e_{L}^{-}$ & $\nu_{e}$ & $h$ \\
\hline 100 & 100 & 7.53 & 0.56 & 0.64 & 0.50 & 0.0031 & 0.0025 & 0.0061 \\
50 & 100 & 7.43 & 0.61 & 0.63 & 0.51 & 0.0031 & 0.0026 & 0.0062 \\
50 & 200 & 7.48 & 0.61 & 0.58 & 0.47 & 0.0028 & 0.0021 & 0.0059 \\
100 & 200 & 7.58 & 0.60 & 0.58 & 0.46 & 0.0028 & 0.0021 & 0.0055 \\
200 & 200 & 7.68 & 0.59 & 0.58 & 0.45 & 0.0028 & 0.0020 & 0.0054 \\
\hline
\end{tabular}

Table 3. Momentum fractions (\%) carried by various parton species at scale $q=100 \mathrm{TeV}$. 


\section{Conclusions}

The energy regime around and beyond the electroweak scale is currently being explored by the LHC experiments, and so far they have found no firm evidence for physics beyond the Standard Model. In the present paper, we have examined the consequences of assuming that the parton distributions of the proton continue to be described by the Standard Model up to very high energies, in the approximation that its symmetries are unbroken above the electroweak scale.

We have implemented numerically the full set of generalized DGLAP evolution equations for all the parton species and interactions of the unbroken SM in leading order. The input PDFs of 5 quark flavors, the gluon, photon and charged leptons at a starting scale $q_{0}=100 \mathrm{GeV}$ for the full SM evolution are obtained from parton and photon PDFs at $10 \mathrm{GeV}$ by QCD plus QED evolution. The input left- and right-handed fermion PDFs are thus identical at scale $q_{0}$ but they evolve differently above that scale. The top quark PDFs (not present in the input) start to evolve from the top mass scale. The input photon is resolved into its $\mathrm{U}(1), \mathrm{SU}(2)$ and mixed components, which are evolved independently from scale $q_{0}$ and reassembled into the photon and transversely polarized $Z^{0}$ at higher scales. The charged and longitudinal vector boson, Higgs and neutrino PDFs are generated dynamically starting from zero at scale $q_{0}$. This simplified treatment misses some symmetry-breaking effects around the electroweak scale, but these are power-suppressed at higher scales and our results should provide a guide to the ways in which the PDFs deviate from pure QCD evolution.

Amongst the most interesting features of the SM is the distinction between left- and right-handed fermions. The evolution of the right-handed PDFs deviates little from pure QCD, owing to the weakness of the U(1) interaction. The left-handed PDFs generally deviate from pure QCD at the $5-10 \%$ level by $10 \mathrm{TeV}$.

Another important SM characteristic is the restoration of isospin symmetry at high scales. This is manifest in the decreasing asymmetry between the up- and down-type quark PDFs, which sets in at $1-10 \mathrm{TeV}$, the up-type being pulled down in the first generation and conversely in the third. The suppression of the asymmetry is a double-logarithmic effect that can be treated in fixed order at present energies but is resummed to all orders in the evolution.

The electroweak bosons are generated quite copiously, the $W^{+}$in particular at high $x$ due to splitting $u \rightarrow d W^{+}$. The photon and $Z^{0}$ PDFs also grow rapidly, eventually exceeding the gluon at high $x$. The PDFs of the longitudinal vector bosons, the Higgs boson and the leptons are generally much smaller as they arise from second-order splittings.

Finally, we have used the generated PDFs to present some parton-parton luminosities at a $100 \mathrm{TeV} p p$ collider. These results are just an illustration of the size of the effects that can be expected at such a future collider, and a more detailed phenomenological analysis will be presented in a forthcoming publication.

In conclusion, we find a rich structure in the proton when probed beyond the electroweak scale. The associated PDFs are interesting and useful in their own right. They also represent a key component of event generators that aim to embody the full Standard Model in initial-state parton showering, a topic we plan to explore further. 


\section{Acknowledgments}

We thank Gavin Salam and Denis Comelli for comments on the manuscript. This work was supported by the Director, Office of Science, Office of High Energy Physics of the U.S. Department of Energy under the Contract No. DE-AC02-05CH11231 (CWB, NF), and partially supported by STFC consolidated grant ST/L000385/1 (BRW).

\section{A Equations used in the forward evolution}

\section{A.1 SU(3) interaction}

- $\mathbf{T}=0$ and $\mathrm{CP}=+$ :

$$
\begin{gathered}
{\left[q \frac{\partial}{\partial q} f_{q}^{0+}\right]_{3}=\frac{\alpha_{3}}{\pi}\left[C_{F} P_{f f, G}^{+} \otimes f_{q}^{0+}+T_{R} P_{f V, G}^{R} \otimes f_{g}\right],} \\
{\left[q \frac{\partial}{\partial q} f_{g}\right]_{3}=\frac{\alpha_{3}}{\pi}\left[C_{A} P_{V V, G}^{+} \otimes f_{g}+C_{F} P_{V f, G}^{R} \otimes f_{\sum_{g}}^{0+}\right] .}
\end{gathered}
$$

Here

$$
f_{\sum_{g}}^{0+}=4 \sum_{q_{L}} f_{q_{L}}^{0+}+2 \sum_{q_{R}} f_{q_{R}}^{0+},
$$

where the sums run over all left-handed quark doublets and all right-handed quarks. The factors of 4 and 2 are due to the different normalizations in eqs. (3.1) and (3.3).

- All other states:

$$
\left[q \frac{\partial}{\partial q} f_{q}\right]_{3}=\frac{\alpha_{3}}{\pi} C_{F} P_{f f, G}^{+} \otimes f_{q}
$$

\section{A.2 U(1) interaction}

- $\mathbf{T}=0$ and $\mathrm{CP}=+$ :

$$
\begin{aligned}
& {\left[q \frac{\partial}{\partial q} f_{f}^{0+}\right]_{1}=\frac{\alpha_{1}}{\pi} Y_{i}^{2}\left[P_{f f, G}^{+} \otimes f_{f}^{0+}+N_{f} P_{f V, G}^{R} \otimes f_{B}\right],} \\
& {\left[q \frac{\partial}{\partial q} f_{B}\right]_{1}=\frac{\alpha_{1}}{\pi}\left[P_{B, 1}^{V} f_{B}+P_{V f, G}^{R} \otimes f_{\sum_{B} f}^{0+}+P_{V H, G}^{R} \otimes f_{H}^{0+}\right],} \\
& {\left[q \frac{\partial}{\partial q} f_{H}^{0+}\right]_{1}=\frac{\alpha_{1}}{\pi} \frac{1}{4}\left[P_{H H, G}^{+} \otimes f_{H}^{0+}+P_{H V, G}^{R} \otimes f_{B}\right],}
\end{aligned}
$$

where

$$
f_{\sum_{B} f}^{0+}=4 \sum_{f_{L}} Y_{f_{L}}^{2} f_{f_{L}}^{0+}+2 \sum_{f_{R}} Y_{f_{R}}^{2} f_{f_{R}}^{0+}
$$

- $\mathbf{T}=1$ and $\mathrm{CP}=+$ :

$$
\left[q \frac{\partial}{\partial q} f_{B W}^{1+}\right]_{1}=\frac{\alpha_{1}}{\pi} \frac{1}{2} P_{B, 1}^{V} f_{B W}^{1+}
$$


- All other states:

$$
\begin{aligned}
& {\left[q \frac{\partial}{\partial q} f_{f}\right]_{1}=\frac{\alpha_{1}}{\pi} Y_{f}^{2} P_{f f, G}^{+} \otimes f_{f}} \\
& {\left[q \frac{\partial}{\partial q} f_{H}\right]_{1}=\frac{\alpha_{1}}{\pi} \frac{1}{4} P_{H H, G}^{+} \otimes f_{H}}
\end{aligned}
$$

\section{A.3 SU(2) interaction}

- $\mathbf{T}=0$ and $\mathrm{CP}=+$ :

$$
\begin{aligned}
& {\left[q \frac{\partial}{\partial q} f_{f_{L}}^{0+}\right]_{2}=\frac{\alpha_{2}}{\pi} \frac{3}{4}\left[P_{f f, G}^{+} \otimes f_{f_{L}}^{0+}+N_{f} P_{f V, G}^{R} \otimes f_{W}^{0+}\right]} \\
& {\left[q \frac{\partial}{\partial q} f_{W}^{0+}\right]_{2}=\frac{\alpha_{2}}{\pi}\left[2 P_{V V, G}^{+} \otimes f_{W}^{0+}+\sum_{f_{L}} P_{V f, G}^{R} \otimes f_{f_{L}}^{0+}+P_{V H, G}^{R} \otimes f_{H}^{0+}\right]} \\
& {\left[q \frac{\partial}{\partial q} f_{H}^{0+}\right]_{2}=\frac{\alpha_{2}}{\pi} \frac{3}{4}\left[P_{H H, G}^{+} \otimes f_{H}^{0+}+P_{H V, G}^{R} \otimes f_{W}^{0+}\right] .}
\end{aligned}
$$

- $\mathbf{T}=0$ and $\mathrm{CP}=-$ :

$$
\begin{aligned}
& {\left[q \frac{\partial}{\partial q} f_{f_{L}}^{0-}\right]_{2}=\frac{\alpha_{2}}{\pi} \frac{3}{4} P_{f f, G}^{+} \otimes f_{f_{L}}^{0-},} \\
& {\left[q \frac{\partial}{\partial q} f_{H}^{0-}\right]_{2}=\frac{\alpha_{2}}{\pi} \frac{3}{4} P_{H H, G}^{+} \otimes f_{H}^{0-} .}
\end{aligned}
$$

- $\mathbf{T}=1$ and $\mathrm{CP}=+$ :

$$
\begin{aligned}
& {\left[\Delta_{f, 2}^{4 / 3} q \frac{\partial}{\partial q} \frac{f_{f_{L}}^{1+}}{\Delta_{f, 2}^{4 / 3}}\right]_{2}=-\frac{\alpha_{2}}{\pi} \frac{1}{4} P_{f f, G}^{+} \otimes f_{f_{L}}^{1+}} \\
& {\left[\Delta_{H, 2}^{4 / 3} q \frac{\partial}{\partial q} \frac{f_{H}^{1+}}{\Delta_{H, 2}^{4 / 3}}\right]_{2}=-\frac{\alpha_{2}}{\pi} \frac{1}{4} P_{H H, G}^{+} \otimes f_{H}^{1+}} \\
& {\left[\Delta_{V, 2}^{1 / 2} q \frac{\partial}{\partial q} \frac{f_{B W}^{1+}}{\Delta_{V, 2}^{1 / 2}}\right]_{2}=0 .}
\end{aligned}
$$

- $\mathbf{T}=1$ and $\mathrm{CP}=-$ :

$$
\begin{aligned}
& {\left[\Delta_{f, 2}^{4 / 3} q \frac{\partial}{\partial q} \frac{f_{f_{L}}^{1-}}{\Delta_{f, 2}^{4 / 3}}\right]_{2}=\frac{\alpha_{2}}{\pi}\left[-\frac{1}{4} P_{f f, G}^{+} \otimes f_{f_{L}}^{1-}+\frac{1}{2} N_{f} P_{f V, G}^{R} \otimes f_{W}^{1-}\right]} \\
& {\left[\Delta_{V, 2}^{1 / 2} q \frac{\partial}{\partial q} \frac{f_{W}^{1-}}{\Delta_{V, 2}^{1 / 2}}\right]_{2}=\frac{\alpha_{2}}{\pi}\left[P_{V V, G}^{+} \otimes f_{W}^{1-}+\sum_{f_{L}} P_{V f} \otimes f_{f_{L}}^{1-}+P_{V H} \otimes f_{H}^{1-}\right]} \\
& {\left[\Delta_{H, 2}^{4 / 3} q \frac{\partial}{\partial q} \frac{f_{H}^{1-}}{\Delta_{H, 2}^{4 / 3}}\right]_{2}=\frac{\alpha_{2}}{\pi}\left[-\frac{1}{4} P_{H H, G}^{+} \otimes f_{H}^{1-}+\frac{1}{2} P_{H V, G} \otimes f_{W}^{1-}\right] .}
\end{aligned}
$$


- $\mathbf{T}=2$ and $\mathrm{CP}=+$ :

$$
\left[\Delta_{V, 2}^{3 / 2} q \frac{\partial}{\partial q} \frac{f_{W}^{2+}}{\Delta_{V, 2}^{3 / 2}}\right]_{2}=-\frac{\alpha_{2}}{\pi} P_{V V}^{+} \otimes f_{W}^{2+} .
$$

\section{A.4 Yukawa interaction}

- $\mathbf{T}=0$ and $\mathrm{CP}=+$ :

$$
\begin{aligned}
& {\left[q \frac{\partial}{\partial q} f_{q_{L}^{3}}^{0+}\right]_{Y}=\frac{\alpha_{Y}}{\pi}\left[P_{q_{L}^{3}, Y}^{V} f_{q_{L}^{3}}^{0+}+P_{f f, Y}^{R} \otimes f_{t_{R}}^{0+}+N_{c} P_{f H, Y} \otimes f_{H}^{0+}\right]} \\
& {\left[q \frac{\partial}{\partial q} f_{t_{R}}^{0+}\right]_{Y}=\frac{\alpha_{Y}}{\pi} 2\left[P_{t_{R}, Y}^{V} f_{t_{R}}^{0+}+P_{f f, Y}^{R} \otimes f_{q_{L}^{3}}^{0+}+N_{C} P_{f H, Y} \otimes f_{H}^{0+}\right]} \\
& {\left[q \frac{\partial}{\partial q} f_{H}^{0+}\right]_{Y}=\frac{\alpha_{Y}}{\pi}\left[P_{H, Y}^{V} f_{H}^{0+}+P_{H f, Y}^{R} \otimes f_{\sum_{H} f}^{0+}\right],}
\end{aligned}
$$

where

$$
f_{\sum_{H} f}^{0+}=f_{t_{R}}^{0+}+f_{q_{L}^{3}}^{0+} .
$$

- $\mathbf{T}=0$ and $\mathrm{CP}=-$ :

$$
\begin{aligned}
& {\left[q \frac{\partial}{\partial q} f_{q^{3}}^{0-}\right]_{Y}=\frac{\alpha_{Y}}{\pi}\left[P_{q_{L}^{3}, Y}^{V} f_{q_{L}^{3}}^{0-}+P_{f f, Y}^{R} \otimes f_{t_{R}}^{0-}-N_{c} P_{f H, Y} \otimes f_{H}^{0-}\right]} \\
& {\left[q \frac{\partial}{\partial q} f_{t_{R}}^{0-}\right]_{Y}=\frac{\alpha_{Y}}{\pi} 2\left[P_{t_{R}, Y}^{V} f_{t_{R}}^{0-}+P_{f f, Y}^{R} \otimes f_{q^{3}}^{0-}+N_{C} P_{f H, Y} \otimes f_{H}^{0-}\right]} \\
& {\left[q \frac{\partial}{\partial q} f_{H}^{0-}\right]_{Y}=\frac{\alpha_{Y}}{\pi}\left[P_{H, Y}^{V} f_{H}^{0-}+P_{H f, Y}^{R} \otimes f_{\sum_{H}}^{0-} f\right]}
\end{aligned}
$$

where

$$
f_{\sum_{H} f}^{0-}=f_{t_{R}}^{0-}-f_{q_{L}^{3}}^{0-} .
$$

- $\mathbf{T}=1$ and $\mathrm{CP}=+$ :

$$
\begin{aligned}
& {\left[q \frac{\partial}{\partial q} f_{q_{L}^{3}}^{1+}\right]_{Y}=\frac{\alpha_{Y}}{\pi}\left[P_{q_{L}^{3}, Y}^{V} f_{q_{L}^{3}}^{1+}-N_{c} P_{f H, Y} \otimes f_{H}^{1+}\right]} \\
& {\left[q \frac{\partial}{\partial q} f_{H}^{1+}\right]_{Y}=\frac{\alpha_{Y}}{\pi}\left[P_{H, Y}^{V} f_{H}^{1+}-P_{H f}^{R} \otimes f_{q_{L}^{3}}^{1+}\right]}
\end{aligned}
$$

- $\mathbf{T}=1$ and $\mathrm{CP}=-$ :

$$
\begin{aligned}
& {\left[q \frac{\partial}{\partial q} f_{t_{L}}^{1-}\right]_{Y}=\frac{\alpha_{Y}}{\pi}\left[P_{t_{L}, Y}^{V} f_{t_{L}}^{1-}+N_{c} P_{f H, Y} \otimes f_{H}^{1-}\right]} \\
& {\left[q \frac{\partial}{\partial q} f_{H}^{1-}\right]_{Y}=\frac{\alpha_{Y}}{\pi}\left[P_{H, Y}^{V} f_{H}^{1-}+P_{H f, Y}^{R} \otimes f_{q_{L}^{3}}^{1-}\right]}
\end{aligned}
$$




\section{A.5 Mixed interaction}

- $\mathbf{T}=1$ and $\mathrm{CP}=+$ :

$$
\begin{aligned}
{\left[q \frac{\partial}{\partial q} f_{f}^{1+}\right]_{M} } & =\frac{\alpha_{M}}{\pi} \frac{Y_{f}}{2} N_{f} P_{f V, G}^{R} \otimes f_{B W}^{1+} \\
{\left[q \frac{\partial}{\partial q} f_{B W}^{1+}\right]_{M} } & =\frac{\alpha_{M}}{\pi}\left[4 \sum_{f_{L}} Y_{f} P_{V f, G}^{R} \otimes f_{f}^{1+}+2 P_{V H, G}^{R} \otimes f_{H}^{1+}\right] \\
{\left[q \frac{\partial}{\partial q} f_{H}^{1+}\right]_{M} } & =\frac{\alpha_{M}}{\pi} \frac{1}{4} P_{H V, G}^{R} \otimes f_{B W}^{1+},
\end{aligned}
$$

Equation (A.37) differs slightly from ref. [8] where, taking into account the definition there of $f_{B 3}=f_{B W} / 2$, an 8 would appear in place of 4 in the first term on the right-hand side.

Open Access. This article is distributed under the terms of the Creative Commons Attribution License (CC-BY 4.0), which permits any use, distribution and reproduction in any medium, provided the original author(s) and source are credited.

\section{References}

[1] A. Buckley et al., General-purpose event generators for LHC physics, Phys. Rept. 504 (2011) 145 [arXiv: 1101.2599] [INSPIRE].

[2] T. Sjöstrand, A Model for Initial State Parton Showers, Phys. Lett. B 157 (1985) 321 [INSPIRE].

[3] R.K. Ellis, W.J. Stirling and B.R. Webber, QCD and collider physics, Camb. Monogr. Part. Phys. Nucl. Phys. Cosmol. 8 (1996) 1 [InSPIRE].

[4] J. Chen, T. Han and B. Tweedie, Electroweak Splitting Functions and High Energy Showering, arXiv:1611.00788 [INSPIRE].

[5] V.N. Gribov and L.N. Lipatov, Deep inelastic e p scattering in perturbation theory, Sov. J. Nucl. Phys. 15 (1972) 438 [inSPIRE].

[6] Y.L. Dokshitzer, Calculation of the Structure Functions for Deep Inelastic Scattering and $e^{+} e^{-}$Annihilation by Perturbation Theory in Quantum Chromodynamics., Sov. Phys. JETP 46 (1977) 641 [INSPIRE].

[7] G. Altarelli and G. Parisi, Asymptotic Freedom in Parton Language, Nucl. Phys. B 126 (1977) 298 [INSPIRE].

[8] P. Ciafaloni and D. Comelli, Electroweak evolution equations, JHEP 11 (2005) 022 [hep-ph/0505047] [INSPIRE].

[9] H. Spiesberger, QED radiative corrections for parton distributions, Phys. Rev. D 52 (1995) 4936 [hep-ph/9412286] [INSPIRE].

[10] A.D. Martin, R.G. Roberts, W.J. Stirling and R.S. Thorne, Parton distributions incorporating QED contributions, Eur. Phys. J. C 39 (2005) 155 [hep-ph/0411040] [INSPIRE].

[11] M. Roth and S. Weinzierl, QED corrections to the evolution of parton distributions, Phys. Lett. B 590 (2004) 190 [hep-ph/0403200] [INSPIRE]. 
[12] NNPDF collaboration, R.D. Ball et al., Parton distributions with QED corrections, Nucl. Phys. B 877 (2013) 290 [arXiv:1308.0598] [InSPIRE].

[13] R. Sadykov, Impact of QED radiative corrections on Parton Distribution Functions, arXiv: 1401.1133 [INSPIRE].

[14] S. Carrazza, Parton distribution functions with QED corrections, arXiv:1509.00209 [INSPIRE].

[15] C. Schmidt, J. Pumplin, D. Stump and C.P. Yuan, CT14QED parton distribution functions from isolated photon production in deep inelastic scattering, Phys. Rev. D 93 (2016) 114015 [arXiv: 1509.02905] [INSPIRE].

[16] A. Manohar, P. Nason, G.P. Salam and G. Zanderighi, How bright is the proton? A precise determination of the photon parton distribution function, Phys. Rev. Lett. 117 (2016) 242002 [arXiv: 1607.04266] [INSPIRE].

[17] P. Ciafaloni and D. Comelli, Sudakov enhancement of electroweak corrections, Phys. Lett. B 446 (1999) 278 [hep-ph/9809321] [INSPIRE].

[18] P. Ciafaloni and D. Comelli, Electroweak Sudakov form-factors and nonfactorizable soft QED effects at NLC energies, Phys. Lett. B 476 (2000) 49 [hep-ph/9910278] [INSPIRE].

[19] M. Ciafaloni, P. Ciafaloni and D. Comelli, Bloch-Nordsieck violating electroweak corrections to inclusive TeV scale hard processes, Phys. Rev. Lett. 84 (2000) 4810 [hep-ph/0001142] [INSPIRE].

[20] M. Ciafaloni, P. Ciafaloni and D. Comelli, Electroweak double logarithms in inclusive observables for a generic initial state, Phys. Lett. B 501 (2001) 216 [hep-ph/0007096] [INSPIRE].

[21] M. Ciafaloni, P. Ciafaloni and D. Comelli, Electroweak Bloch-Nordsieck violation at the TeV scale: 'Strong' weak interactions?, Nucl. Phys. B 589 (2000) 359 [hep-ph/0004071] [INSPIRE].

[22] M. Ciafaloni, P. Ciafaloni and D. Comelli, Enhanced electroweak corrections to inclusive boson fusion processes at the TeV scale, Nucl. Phys. B 613 (2001) 382 [hep-ph/0103316] [INSPIRE].

[23] M. Ciafaloni, P. Ciafaloni and D. Comelli, Towards collinear evolution equations in electroweak theory, Phys. Rev. Lett. 88 (2002) 102001 [hep-ph/0111109] [INSPIRE].

[24] P. Ciafaloni, D. Comelli and A. Vergine, Sudakov electroweak effects in transversely polarized beams, JHEP 07 (2004) 039 [hep-ph/0311260] [INSPIRE].

[25] P. Ciafaloni and D. Comelli, The Importance of weak bosons emission at LHC, JHEP 09 (2006) 055 [hep-ph/0604070] [INSPIRE].

[26] M. Ciafaloni, P. Ciafaloni and D. Comelli, Electroweak double-logs at small x, JHEP 05 (2008) 039 [arXiv:0802.0168] [INSPIRE].

[27] P. Ciafaloni and A. Urbano, Infrared weak corrections to strongly interacting gauge bosons scattering, Phys. Rev. D 81 (2010) 085033 [arXiv:0902.1855] [INSPIRE].

[28] P. Ciafaloni, D. Comelli, A. Riotto, F. Sala, A. Strumia and A. Urbano, Weak Corrections are Relevant for Dark Matter Indirect Detection, JCAP 03 (2011) 019 [arXiv:1009.0224] [INSPIRE]. 
[29] S. Forte et al., The Standard Model from LHC to future colliders, Eur. Phys. J. C 75 (2015) 554 [arXiv: 1505.01279] [INSPIRE].

[30] M.L. Mangano et al., Physics at a $100 \mathrm{TeV}$ pp collider: Standard Model processes, CERN Yellow Report (2017) 1 [arXiv:1607.01831] [INSPIRE].

[31] J. Butterworth et al., PDF 4 LHC recommendations for LHC Run II, J. Phys. G 43 (2016) 023001 [arXiv: 1510.03865] [INSPIRE]. 